\title{
Substrate properties of zebrafish Rtn4b/Nogo and axon regeneration in the zebrafish optic nerve
}

\author{
Vsevolod Bodrikov $^{1 *}$ | Cornelia Welte ${ }^{1 *}$ | Marianne Wiechers ${ }^{1 *}$ | \\ Markus Weschenfelder ${ }^{2}$ | Gurjot Kaur ${ }^{1}$ | Aleksandra Shypitsyna ${ }^{1}$ | \\ Alejandro Pinzon-Olejua ${ }^{1}$ | Martin Bastmeyer ${ }^{2}$ | Claudia A. O. Stuermer ${ }^{1}$
}

${ }^{1}$ Department of Biology, University of Konstanz, Konstanz, Germany

${ }^{2}$ Zoological Institute, Cell and Neurobiology Biology, Karlsruhe Institute of Technology (KIT), Karlsruhe, Germany

\section{Correspondence}

Claudia A. O. Stuermer, Department of

Biology, University of Konstanz,

Universitätsstr. 10, 78456 Konstanz,

Germany.

Email: Claudia.stuermer@uni-konstanz.de

Present addresses

Aleksandra Shypitsyna, European Bioinformatics Institute, Cambridge, United Kingdom

Alejandro PinzonOlejua, Max Planck Institute for Brain Research, Max-von-Laue-Str. 4 60438, Frankfurt am Main, Germany

\begin{abstract}
This study explored why lesioned retinal ganglion cell (RGC) axons regenerate successfully in the zebrafish optic nerve despite the presence of Rtn4b, the homologue of the rat neurite growth inhibitor RTN4-A/Nogo-A. Rat Nogo-A and zebrafish Rtn4b possess characteristic motifs (M1-4) in the Nogo-A-specific region, which contains delta20, the most inhibitory region of rat Nogo-A. To determine whether zebrafish M1-4 is inhibitory as rat M1-4 and Nogo-A delta20, proteins were recombinantly expressed and used as substrates for zebrafish single cell RGCs, mouse hippocampal neurons and goldfish, zebrafish and chick retinal explants. When offered as homogenous substrates, neurites of hippocampal neurons and of zebrafish single cell RGCs were inhibited by zebrafish M1-4, rat M1-4, and Nogo-A delta20. Neurite length increased when zebrafish single cell RGCs were treated with receptor-type-specific antagonists and, respectively, with morpholinos (MO) against S1PR2 and S1PR5a-which represent candidate zebrafish Nogo-A receptors. In a stripe assay, however, where M1-4 lanes alternate with polylysine-(Plys)-only lanes, RGC axons from goldfish, zebrafish, and chick retinal explants avoided rat M1-4 but freely crossed zebrafish M1-4 lanes-suggesting that zebrafish M1-4 is growth permissive and less inhibitory than rat M14. Moreover, immunostainings and dot blots of optic nerve and myelin showed that expression of Rtn4b is very low in tissue and myelin at 3-5 days after lesion when axons regenerate. Thus, Rtn4b seems to represent no major obstacle for axon regeneration in vivo because it is less inhibitory for RGC axons from retina explants, and because of its low abundance.
\end{abstract}

\section{KEYWORDS}

growth in stripes, neurite growth inhibitor, Nogo-A/RTN4-A homologue, Nogo receptors, optic nerve regeneration, retinal explants, single cell neurons, zebrafish Rtn4b, RRID: AB_10562367, RRID:AB_10000211, RRID:AB_2636940, RRID:AB_2636941, RRID:AB_2315032, RRID: AB_301787

\section{1 | INTRODUCTION}

Neurons in the central nervous system (CNS) of mammals fail to regenerate axons after injuries due to inhibitory components in their environment and intrinsic conditions which reduce the growth capacity. In the CNS of fishes, however, axons do regenerate and restore functional connections due to a growth-permissive environment and

*The first three authors contributed equally to the study neuron intrinsic properties which favor growth (Stuermer, 2010; Stuermer, Bastmeyer, Bahr, Strobel, \& Paschke, 1992). A major impediment of axon regeneration in mammals is CNS myelin and myelinassociated axon growth inhibitors (Schwab, 2010) which can even block the elongation of fish axons (Bastmeyer, Beckmann, Schwab, \& Stuermer, 1991). The major inhibitor in mammalian CNS myelin is Nogo-A/RTN4-A, the longest transcript of the reticulon (rtn)-4 gene (Schwab, 2010). Nogo-A has two inhibitory stretches: Nogo-66 in the C-terminal reticulon homology domain (RHD) and delta20 in the so- 
called Nogo-A specific N-terminal region (NSR, Figure 1; Shypitsyna, Malaga-Trillo, Reuter, \& Stuermer, 2011). The receptor for Nogo-66 is Nogo-receptor 1 (NgR1) in complex with accessory proteins (Fournier, GrandPre, \& Strittmatter, 2001; Mandemakers \& Barres, 2005) whereas Nogo-A delta20 binds to the G-protein coupled receptor (GPCR) sphinosine-1-phosphate receptor 2 (S1PR2), which is associated with tetraspanin and other (unpublished) co-receptors (Kempf et al., 2014; Thiede-Stan \& Schwab, 2015).

How does this compare to the situation in fish? In contrast to mammals, fish CNS myelin is comparatively growth permissive. This was shown by experiments using either optic nerve tissue sections or myelin fractions from the fish in comparison to sections and myelin from the rodent CNS. In these assays, fish CNS myelin was crossed by axons (Bastmeyer et al., 1991; Carbonetto, Evans, \& Cochard, 1987; Vanselow, Schwab, \& Thanos, 1990) whereas mammalian CNS myelin inhibited growth of fish retinal ganglion cell (RGC) axons as well as mammalian DRG axons (Caroni \& Schwab, 1988; Wanner et al., 1995). However, it has more recently been recognized that zebrafish possess RTN4-A/Nogo-A homologues, namely Rtn4a and Rtn4b (rtn-6) (Diekmann et al., 2005; Shypitsyna et al., 2011) which are widely expressed in the zebrafish CNS (Pinzon-Olejua, Welte, Abdesselem, Malaga-Trillo, \& Stuermer, 2014) including optic nerve and CNS myelin (Welte, Engel, \& Stuermer, 2015). Both proteins have a conserved C-terminal reticulon homology domain (RHD), the hallmark of this gene family (Diekmann et al., 2005; Oertle et al., 2003). A comparative study revealed that the Nogo-66 region is highly conserved and present in the RHD of zebrafish Rtn4a. Nogo-66 binding to the Nogo receptor ( $\mathrm{NgR}$ ) surprisingly promotes neurite growth (Abdesselem, Shypitsyna, Solis, Bodrikov, \& Stuermer, 2009) whereas rat Nogo-66 and NgR-mediated signal transduction inhibits growth (Fournier et al., 2001). The N-terminal regions of zebrafish Rtn4a and -b differ from each other (Shypitsyna et al., 2011). The N-terminus of zebrafish Rtn4a is short (Diekmann et al., 2005) and is homologous to the RTN4B isoform (Shypitsyna et al., 2011), whereas the $N$-terminus of zebrafish Rtn4b shares some similarity with rat Nogo-A/RTN4-A (Figure 1; Shypitsyna et al., 2011). In particular, four short motifs-termed M1-4-were found to be conserved between fish Rtn $4 \mathrm{~b}$ in several teleost species and mammalian RTN4-A (Figure 1a). Three of these motifs are located within the delta20 region which is the most inhibitory portion of the mammalian RTN4-A/Nogo-A protein (Oertle et al., 2003; Schwab, 2010; Shypitsyna et al., 2011). M1 lies outside of the delta20 region at the $\mathrm{N}$ terminal side. The motifs M1, M3, and M4 are conserved in zebrafish whereas the M2 region does not fully match the motif profile (Shypitsyna et al., 2011) (Figure 1a). For recombinant protein expression we chose a region comprising M1-4 in the rat and the corresponding region in the zebrafish gene which we term M1-4. We anticipated that rat M1-4 is as inhibitory (or more) as delta20, and asked whether M14 of zebrafish Rtn4b may likewise be inhibitory. Moreover, zebrafish possess members of the S1PR family (Gu, Forostyan, Sabbadini, \& Rosenblatt, 2011; Kai, Heisenberg, \& Tada, 2008; Matsui et al., 2007) which represent putative Nogo-A specific receptors (Kempf et al., 2014). These receptors could transmit growth-inhibiting signals triggered by delta20 or M1-4 binding to growth cones provided that the neurons (such as RGCs) express the relevant receptors. If so, how could this be reconciled with the older data showing growth permissiveness of fish CNS myelin and optic nerve tissue? This was addressed in a series of experiments in the present study.

To examine whether rat M1-4 and the zebrafish M1-4 region are inhibitory to axon growth, like rat Nogo-A delta20, we generated and recombinantly expressed rat delta20, rat M1-4 and zebrafish M1-4. We compared axon growth on delta20, rat M1-4 and zebrafish M1-4 as the sole homogeneous substrate. We also determined axon behavior on striped substrates (Vielmetter, Stolze, Bonhoeffer, \& Stuermer, 1990) containing alternating lanes of rat M1-4 versus polylysine, and zebrafish M1-4 versus polylysine, respectively. If M1-4 would be inhibitory, axons from retina explants are expected to avoid the M1-4 containing stripes and to elongate preferentially on polylysine. Inhibition by rat or zebrafish M1-4 requires the presence of the receptors. We show expression of S1PR2 and S1PR5a in zebrafish RGCs which mediate the inhibition by M1-4. Also, we repeated tests of the substrate properties of zebrafish and rat CNS myelin. The outcome of these assays confirmed earlier results: fish CNS myelin was significantly more permissive than rat CNS myelin (Bastmeyer et al., 1991; Carbonetto et al., 1987; Caroni \& Schwab, 1988; Vanselow et al., 1990; Wanner et al., 1995). This led to the next experiment: we determined the expression and exposure of Rtn4b M1-4 during the time that axons cross the lesion and regenerate into the brain-side portion of the optic nerve. Our results show that zebrafish optic nerve myelin at 5 days after optic nerve section (ONS) as well as nerve homogenates at 3 days after ONS contain significantly less Rtn4b than non-transected (normal) nerves. The amount of the myelin protein PO was nearly the same in transected versus normal nerves and was, in fact, used as standard against which Rtn4b was determined. Thus, zebrafish Rtn4b is less inhibitory for RGC growth cones from retinal explants and is very low in optic nerve myelin after ONS and during axon regeneration.

\section{I MATERIAL AND METHODS}

\section{$2.1 \mid$ Animals}

Adult goldfish and zebrafish were raised in the animal facility of the University of Konstanz. Optic nerve lesion was performed under MS222 anesthesia in compliance with animal welfare legislation of the state Baden-Württemberg (No G13/98).

\section{2 | Explant cultures from chick}

Retinae of E6-E7 chick embryos were dissected in ice-cold Hanks' medium, placed on nitrocellulose filters and cut into $250 \mu \mathrm{m}$ wide stripes. Explants were placed on the stripe substrates and grown in F12 medium containing $0.4 \%$ methylcellulose, $2 \%$ chicken, and $5 \%$ fetal calf serum. After 20-24 hr, the cultures were fixed and stained with Alexa-488-phalloidin (Invitrogen/Thermo Fisher, see below). 


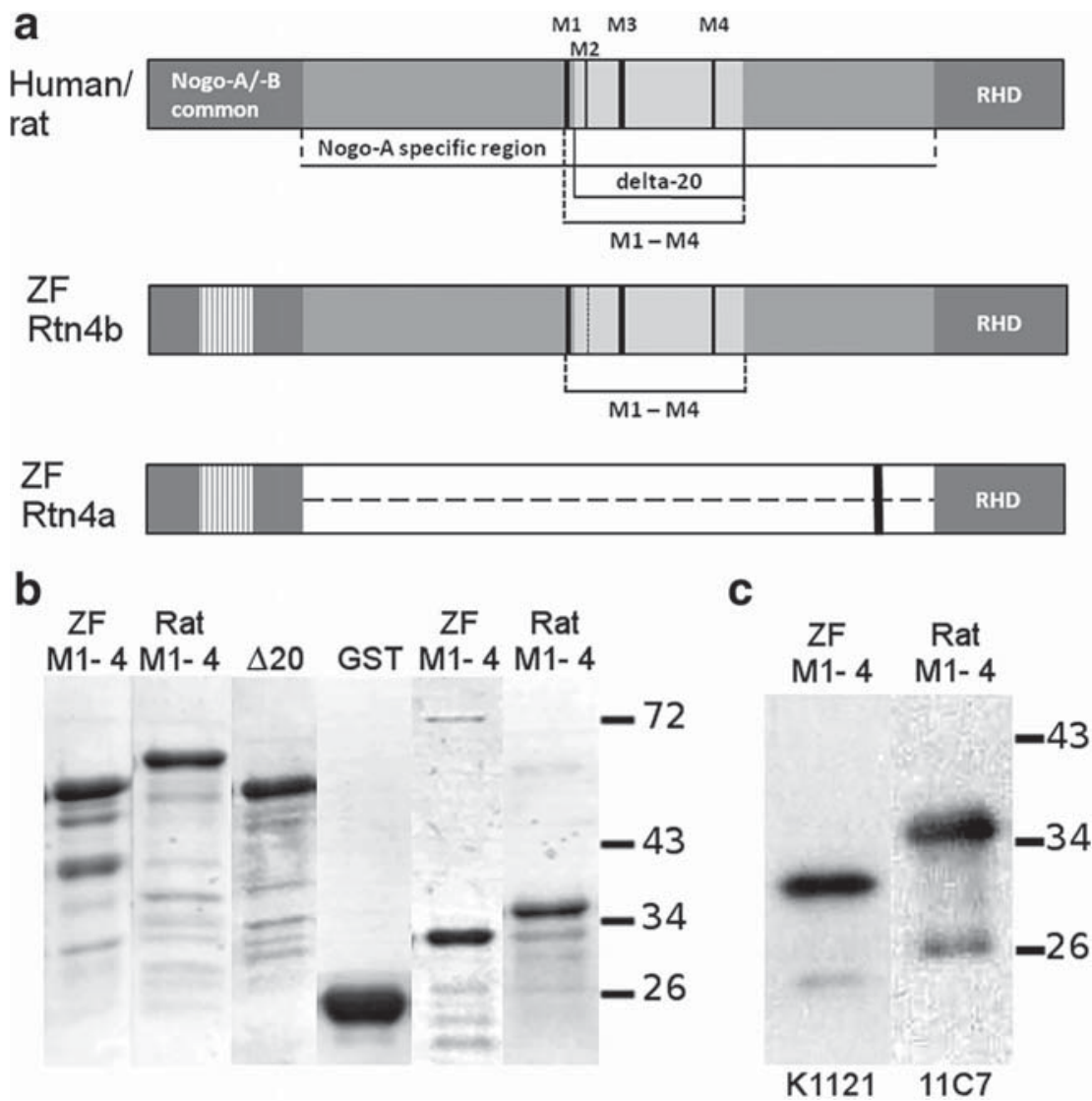

FIGURE 1 Comparison of Nogo genes and generation of recombinantly expressed M1-4 proteins. (a) Schematic representation of human/ rat RTN4A/Nogo-A with the C-terminal Reticulon homology domain (RHD) and the N-terminal Nogo-A/-B common region, next to the Nogo-A specific region. The position of the motifs M1, M2, M3, and M4, the region used for recombinant expression (M1-4) as well as the location of the most inhibitory delta20 region are indicated (Shypitsyna et al., 2011). Zebrafish (ZF) Rtn4b and Rtn4a possess the RHD. Rtn4b has a long N-terminal Rtn4b specific region with motifs M 1,3,4 in similar positions as in the human/rat RTN4-A gene. M2 does not fully match the motif profile (dotted line) but is highly conserved in other teleost species. ZF Rtn4a mainly consists of the RHD and a short $\mathrm{N}$-terminal stretch which is homologous to the RTN4B variable part. (b) Coomassie-stained gels with recombinantly expressed Zebrafish (ZF) M1-4-GST, Rat M1-4-GST, Nogo-A delta20-GST and GST alone, ZF M1-4 and Rat M1-4 after cleavage of GST. The molecular weight markers (in $\mathrm{kDa}$ ) are shown to the right. (c) In Western blots with recombinantly expressed rat M1-4 and after cleavage of GST, mAb 11C7 recognizes the M1-4 protein. Recombinantly expressed and GST-cleaved ZF M1-4 is recognized by the pAB K1121 against Rtn4b

\section{3 | Stripe and outgrowth assays}

Zebrafish and goldfish retinae were isolated between 7 and 14 days after ONS and cut into $300 \mu \mathrm{m}$ wide strips as described (Vielmetter \& Stuermer, 1989). A special silicon matrix was used to apply peptides M1-4 and GST in stripes onto poly-L-lysine (Plys)-coated coverslips (Oertle et al., 2003; Vielmetter et al., 1990; Weschenfelder, Weth, Knoll, \& Bastmeyer, 2013) at concentrations of 20-30 $\mu$ M. Retina explants were placed perpendicular onto the striped substrate and incubated at the species-specific optimal temperature for 2-4 days in F12 medium with $1 \%$ FCS. Axons from retinal explants were photographed live under phase contrast optics at an inverted microscope. Cultures were fixed, permeabilized (4\% PFA [paraformaldehyde] and $0.1 \%$ Triton) and labeled with Alexa-coupled Phalloidin. Immunostainings with mAB $11 C 7$ (Oertle et al., 2003; kindly provided by Martin Schwab, Zurich, Switzerland) and our pAB K1121 (see below) against zebrafish M1-4 (Pinzon-Olejua et al., 2014; Welte et al., 2015) was used to visualize the substrate stripes after fixation at a fluorescence microscope (Axioplan 2, Zeiss Jena, Germany). The evaluation of the stripes was done by a person blinded to the origin (fish or rat) of the M1-4 proteins and whether stripes contained GST or M1-4.

\section{4 | Zebrafish single cell RGC culture and transfection}

Single RGCs were isolated from zebrafish retinae and maintained on Poly-D-lysine (PDL)-coated coverslips in L15 medium (Sigma-Aldrich, St Louis, MO) supplemented with $0.5 \%$ FCS, $1 \%$ Penicillin/Streptomycin, $0.5 \mathrm{mM}$ glutamine and $5 \mu \mathrm{g} / \mathrm{ml}$ basic fibroblast growth factor (bFGF, all Invitrogen/Thermo Fisher Carlsbad, CA). Cells were grown at $28^{\circ} \mathrm{C}$ and $5 \% \mathrm{CO}_{2}$. After 3 days in culture they were transfected with $(1 \mu \mathrm{M})$ morpholinos (MO) against S1PR2 and S1PR5a and control MO (contr $\mathrm{MO}$ ), respectively (see below) with the Endo-Porter transfection reagent (Gene Tools, Philomath, OR). 
Cells were fixed in 4\% PFA in PBS, stained with Alexa-Fluor488-Phalloidin (Life Technologies/Thermo Fisher, A11017; RRID: $A B_{-}$10562367) or Alexa-Fluor-568 Phalloidin (A12380, Invitrogen/ Thermo Fisher) and DAPI (4',6-diamidino-2-phenylindole, $100 \mathrm{ng} / \mathrm{ml}$, Sigma-Aldrich) for $2 \mathrm{hr}$ at RT and mounted with Mowiol (Hoechst, Frankfurt, Germany) on coverslips for epifluorescence analysis using a 40x objective and the Axioplan2 microscope (Zeiss). Cells containing the Lissamine-tagged MOs (Gene Tools, Philomath, OR) in the perinuclear cytoplasm were identified and the length of the longest neurite (longer than the cell diameter) was measured with ImageJ (NIH ImageJ software). Recombinant proteins were used at concentration $200 \mathrm{nM}$ (with GST) or 600 nM (after GST cleavage).

\section{5 | Culture of hippocampal neurons}

Hippocampal neurons from 1 to 2 days old wt C57BL/6J mice were exposed to papain (Sigma-Aldrich) $30 \mathrm{~min}, 37^{\circ} \mathrm{C}$, centrifuged $(80 \times \mathrm{g}, 5$ min, $37^{\circ} \mathrm{C}$ ), resuspended and seeded on PDL coated coverslips (12-well plates, Neurobasal A with B-27 serum-free supplement, Life Technologies/Thermo Fisher, and $5 \mu \mathrm{g} / \mathrm{ml} \mathrm{bFGF).} \mathrm{After} 3$ days, FGF was increased $(10 \mu \mathrm{g} / \mathrm{ml})$. Cells grew at $37^{\circ} \mathrm{C}$ and $5 \% \mathrm{CO}_{2}$ (Bodrikov, Solis, $\&$ Stuermer, 2011). After fixation, neurons ( 48 or $96 \mathrm{hr}$ ) on glass coverslips were stained with Alexa-488-phalloidin to measure neurite length (NIH ImageJ software).

\subsection{Cloning of zebrafish rtn4b M1-4 specific region into a GST expression vector}

The rtn4b-M1-M4 region (Shypitsyna et al., 2011) was amplified by PCR from a TOPO2.1 vector containing the rtn4b ORF (Pinzon-Olejua et al., 2014). Forward (FW) 5'-GGGAATTCT-AGCCCGTCTCCAGACCTGCTCCAGGA-3' and reverse (RV) 5'-GGGTCGACCTA-CTGCAGACCCTGGAGCAGCTCTGCC- $3^{\prime}$ primers containing EcoRI and Sall restriction sites were designed to amplify 490 base pairs including the M1 to M4 motifs. The PCR product was digested with EcoRI and Sall and cloned in frame after the thrombin cleavage site of the pGEX-4T-3 GST expression vector (GE Healthcare, Braunschweig, Germany). All the above-mentioned PCR reactions were performed with the Phusion high-fidelity DNA polymerase (Finnzymes/Thermo Fisher) and positive clones were further sequenced.

\subsection{Subcloning of rat delta20 and cloning of rat M1-4}

The pET28 expression vector containing the Rat-NIG $\Delta 20$ construct was kindly provided by M. E. Schwab (University and ETH Zürich, Switzerland). The delta20 sequence was PCR-amplified from this vector and inserted into the pGEX-KG plasmid.

To create the recombinant Rat M1-4 protein, the original pGEX- $\Delta 20$ plasmid was used as template for elongation by PCR with FW primer $5^{\prime}$ AAAGGATCCACGCCAGATTTAGTTCAGGAAGCATGTGAAAGTGAACTGAATGAAGCCACAGGTACAAAGATTGCTTATGAAACAAAAGTGG$3^{\prime}$ and RV primer 5'-GAGAGCTTTGTTTCTITAATTAAATCACACGCAATGGATA TATAAGGAG-3' containing BamHI and Pacl restriction sites. PCR product was digested with the respective enzymes and cloned in frame behind the thrombin cleavage site of the pGEX-4T-3 GST expression vector (GE Healthcare).

\subsection{Protein production and purification}

To produce rat M1-4 and delta20 and zebrafish M1-4, overnight cultures with the transformed BL21-CodonPlus (DE3)-RIPL cells were incubated at $37^{\circ} \mathrm{C}$ and $230 \mathrm{rpm}$ on the bacterial shaker. When cell density reached a value of 0.5 , IPTG was added to the final concentration of $1 \mathrm{mM}$. Four hours later, cells were centrifuged at 7,000 rpm for 20 min at $4^{\circ} \mathrm{C}$. The cells were resuspended in $20 \mathrm{ml}$ lysis buffer (PBS, DTT $10 \mathrm{mM}$, EDTA $2 \mathrm{mM}$ ) supplemented with protease inhibitors and stored at $-20^{\circ} \mathrm{C}$. Cell aliquots were defrozen and lyzed by sonification. The lysates were centrifuged at 14,000 rpm.

All recombinant proteins were purified via affinity chromatography over glutathione sepharose (GST) beads in the batch/column method according to the manufacturer's recommendations (GST, Gene Fusion System, GE Healthcare). One milliliter of beads was equilibrated with lysis buffer. To elute the proteins from the matrix, elution buffer (containing reduced L-glutathion) was added to the columns and the beads were incubated for $30 \mathrm{~min}$. All flow-through fractions were collected and analyzed by SDS-PAGE. Protein concentration of the elution fractions was measured by Bradford reaction.

For the stripe assay, the GST was cleaved from the M1-4 peptides by thrombin ( $20 \mathrm{U}, 3-4 \mathrm{hr}$ ) according to the manufacturers instruction (Sigma-Aldrich). M1-4 peptides were quality-controlled in Commassie gels and used at concentrations between 50 and $90 \mu \mathrm{M}$. For evaluation of protein expression, solubility and efficiency of purification, the samples were analyzed on SDS-PAGE and Western blots.

\section{9 | Antibody characterization}

$11 \mathrm{C} 7$ is a monoclonal $A B$ from mouse against rat Nogo-A raised against recombinantly expressed His-tagged Nogo-A delta2O (Oertle et al., 2003) which was kindly provided by M. E. Schwab, University and ETH Zurich; Zurich; Switzerland (Cat\# Nogo-A, RRID:AB_10000211). It recognized the peptide against which it was raised and $210 \mathrm{kDa}$ Nogo-A in Western blots with lysates of cultured oligodendrocytes (Oertle et al., 2003) and binds to GST-delta20 and GST-M1-4 as well as to delta20 and M1-4 after cleavage of GST in our present study (Figure 1). pAB IK964 is a polyclonal AB from a rabbit which was immunized with the His-tagged Nogo-66 peptide of zebrafish Rtn4a (Abdesselem et al., 2009; Pinzon-Olejua et al., 2014; Claudia Stuermer Lab Universität Konstanz; Germany Cat\# Rtn4a, RRID:AB_2636940). The specificity was shown in lysates of zebrafish embryos after injections of Rtn4a-specific and control morpholinos, respectively, into the yolk at early stages of development, and by immunostainings at increasing developmental stages as well as in the adult optic nerve after lesion (Abdesselem et al., 2009). pAB K1121 was raised in a rabbit against zebrafish M1-4 of Rtn4b after cleavage of GST (Pinzon-Olejua et al., 2014; Welte et al., 2015; Claudia Stuermer Lab, Universität Konstanz; Germany Cat\# Rtn4b, RRID:AB_2636941). The serum was affinity-purified against the peptide and recognizes the 
antigen against which it was raised as well as $180 \mathrm{kDa}$ in Western blots with lysates of zebrafish embryos and brain (Pinzon-Olejua et al., 2014; Welte et al., 2015). The signal in Western blots with lysates of the retina is reduced after application of Rtn $4 b$ specific MOs to the cut optic nerve (Welte et al., 2015). The pAB against Rtn4b binds to tissue sections through the optic nerve in a previous (Welte et al., 2015) and the present study. pAB against zebrafish PO was kindly provided by Edward A. Burton (University of Pittsburgh, USA: Bai 519: 1580, 2011 Cat\# PO (peptide 2), RRID:AB_2315032). It was raised against recombinantly expressed peptides from $\mathrm{PO}$ in rabbits and immuno-affinity purified. It recognizes the $23 \mathrm{kDa}$ PO protein in Western blots and stains myelin in tissue sections through the zebrafish brain and optic nerve (Bai, Sun, Stolz, \& Burton, 2011). The anti- $\alpha$-Tubulin $A B$ (15246) was purchased from ABCAM (Cambridge, UK, RRID: AB_301787). It was raised in rabbits which were immunized with key hole limpet (KLH) conjugated synthetic peptide corresponding to the $C$ terminal amino acid $426-450$ of human $\alpha$-tubulin. It recognizes the antigen in many species including zebrafish which was approved in our present study.

\section{$2.10 \mid$ Immunostaining of cryosections}

For cryosections, the optic nerves were isolated, transferred directly into TissueTec (Sakura, Finetek Europe) at $-20^{\circ} \mathrm{C}$ and cut on a cryostat. The $10 \mu \mathrm{m}$ thick sections were transferred to Plys-coated slides and allowed to dry, and either stored at $-20^{\circ} \mathrm{C}$ or subjected directly to immunostainings with Rtn4b pAB (K1121), pAB against Rtn4a (IK 964; Abdesselem et al., 2009; Pinzon-Olejua et al., 2014; Welte et al., 2015), and anti-PO pAB. After washes in PBS, sections were coverslipped with Mowiol (Calbiochem/Merck, Darmstadt, Germany). Images were acquired at a confocal laser-scanning microscope (LSM700 META; Zeiss, Jena, Germany) with a 20x lens.

\section{$2.11 \mid$ Dot blots and Western blots}

Isolated zebrafish optic nerves were lysed in RIPA-buffer. The evaluation of Rtn4a and Rtn4b in optic nerve/tract myelin, normal and after ONS, was done in dot blots. The signals with the ABs against Rtn4a (IK 964) and Rtn4b (K1121) were compared to PO pAB. Homogenates of the optic nerve were prepared in ice-cold PBS buffer supplied with EDTA-free inhibitors. Myelin fractions were prepared as described below. Two microliters of homogenates or myelin samples of the same optical density were loaded to the blot membrane. The intensity of protein dots was determined by ImageJ, the Rtn $4 b$ dot was normalized to PO control dots and statistically evaluated using the Student's $t$-test. For Western blot analysis, isolated zebrafish optic nerves were lysed in RIPA-buffer. Blots were exposed to pAB K1121 against Rtn4b (diluted $1: 3,000)$ and anti- $\alpha$-Tubulin $A B$. The intensity of protein bands was determined by ImageJ, the Rtn $4 b$ bands normalized to the loading control and statistically evaluated using the Student's t-test.

\subsection{2 | Myelin preparation}

Myelin of zebrafish brain and optic nerves, and of rat spinal cord/hindbrain was prepared as previously described (Bastmeyer et al., 1991;
Caroni \& Schwab, 1988). Briefly, zebrafish brain and optic nerves and adult rat spinal cords/hindbrain were rapidly dissected free in ice-cold PBS and transferred to homogenization buffer (10 mM Tris- $\mathrm{HCl}, 2 \mathrm{mM}$ $\mathrm{CaCl}, 1 \mathrm{mM}$ EDTA, $1 \mathrm{mM}$ spermidine, $5 \mathrm{mM}$ iodoacetamite, and Protease inhibitor complete (Roche Diagnostics, Basel, Switzerland). After homogenization, fractions enriched in myelin were obtained through centrifugation at $23,000 \mathrm{rpm}$ for $12 \mathrm{~min}$ at $4^{\circ} \mathrm{C}$ in a sucrose step gradient (20 and $50 \%$ sucrose for zebrafish brain and optic nerve myelin and 15,25 , and $50 \%$ sucrose for rat spinal cord/hindbrain myelin, all in homogenization buffer; modified after Colman, Kreibich, Frey, \& Sabatini, 1982). Myelin-enriched fractions were washed three times at $4^{\circ} \mathrm{C}$ in PBS containing protease inhibitors and frozen at $-20^{\circ} \mathrm{C}$. Myelin was dropped through a pipette onto PDL-coated coverslips and let to dry (30 min at room temperature, RT) resulting in circular areas of 300$600 \mu \mathrm{m}$ diameter. Isolated zebrafish single cell RGCs and mouse hippocampal neurons were given onto the coverslip with myelin dots and cultivated at species-specific temperature and culture conditions. Four to five days later, cells were fixed and stained with phalloidin and DAPI to visualize cells and cell processes.

\subsection{S1PR2 and S1PR5a morpholinos and application}

Morpholinos (MO) against S1PR2 and S1PR5a were used at a concentration of either 0.5 or $1 \mu \mathrm{M}$ and applied to cultured single cell RGCs. The MOs against S1PR2 and S1PR5a were: 5'-CCGCAAACAGACGGCAAGTAGTCAT-3' (position on mRNA 1/25 (Matsui et al., 2007)) and $5^{\prime}$-AGCTTCCATTTCACTTCTGCTGACC-3' (position on mRNA $-16 / 9)$. The control MO sequence was: $5^{\prime}$-aacgaacgaacgaacgaacgaacgc- $3^{\prime}$ (absent from the zebrafish genome).

\subsection{4 | S1PR2 and S1PR5a; reverse transcription (RT)- PCR}

Adult zebrafish retina were homogenized in TRIzol reagent, and total RNA was isolated by Trizol RNA isolation protocol using phase separation. Total RNA ( $1 \mu \mathrm{g})$ was used to generate cDNA using Reverse transcriptase and oligodT20 or random hexamer primers (SuperScript ${ }^{\circledR}$ III First- Strand Synthesis System, Invitrogen/Thermo Fisher). The PCR was performed with Taq polymerase and cycle conditions were $95^{\circ} \mathrm{C}$ for $30 \mathrm{~s}$ followed by 30 cycles at $95^{\circ} \mathrm{C}$ for $20 \mathrm{~s}$, gradient $48-68^{\circ} \mathrm{C}$ for $30 \mathrm{~s}, 68^{\circ} \mathrm{C}$ for $90 \mathrm{~s}$ and $68^{\circ} \mathrm{C}$ for $5 \mathrm{~min}$. Product was analyzed on Ethidium bromide containing $1 \%$ agarose gels. Primer sequences for RT-PCR are as follows:

S1PR2 FW:ATGACTACTTGCCGTCTGTTTGCGG, RV:TAGAATT CTCAAACACAGGTAGTGCAGTCCTGC;

S1PR5a FW:ATGGAAGCTTCATACGCTGCCAGTG,

RV: TACTCGAGTCAACTCTTAAGTATCATTGGGTAA;

Rtn4b FW: CCGTCTCCAGACCTGCTCCAGGA

RV: GGCAGAGCTGCTCCAGGGTCTGCA (Rtn4b was used as control). 


\section{3 | INHIBITORS}

Inhibitors of S1PR2 and S1PR1,3-5 were the commercially available substances JTE-013 (Tocris Bioscience, Bristol, UK) and FTY720 (Cayman/Biomol, Hamburg, Germany) (Rosen, Gonzalez-Cabrera, Sanna, \& Brown, 2009) which were dissolved in DMSO and applied to single cell RGCs.

\section{1 | In situ hybridization (ISH)}

ISH was performed with digoxigenin-labeled probes using MAXIscript T7/T3 Transcription (Thermo Fischer). Sense and anti-sense probes were prepared from PCR products of S1PR2 (NM_001159970) and S1PR5a (NM_001007316) ligated into pBluscriptKS

(S1PR2 FW: TATTCTAGA CTCAACACCC GATACTACATCCGG,

RV: ATAGTCGACCCAACACAGGTAGTGCAGTCCTG, S1PR5a FW: TATTCTAGATATTCCGCATCGTCAAGTCCA,

RV: ATAGTCGACCCACTCTTAAGTATCCTTGGGT) transcribed using $\mathrm{t} 7$ (for sense) or $\mathrm{t} 3$ (for anti-sense) RNA polymerase. Tissue preparation for ISH: whole eyes were fixed overnight at $4^{\circ} \mathrm{C}$ in $4 \%$ PFA in $0.1 \mathrm{M}$ phosphate buffer, $\mathrm{pH} 7.4$, cryoprotected in phosphate buffered $20 \%$ sucrose overnight at $4^{\circ} \mathrm{C}$ and embedded with tissue-Tek O.C.T compound (Sakura Finetek, Torrance, CA). Embedded samples were kept frozen at $-20^{\circ} \mathrm{C}$ until sectioned to $10 \mu \mathrm{m}$ on a cryostat. Sections were collected on Superfrost Ultra plus adhesion slides, dried for 20 min at RT and directly taken for ISH procedure modified after Saul, Koke, and Garcia (2010): dried retina cross sections were post-fixed with 4\% PFA in DEPC (Sigma Aldrich) treated 1X PBS (5 min, RT) and incubated once in $0.01 \%$ DEPC activated PBS for $10 \mathrm{~min}$. Cross sections were partially digested with proteinase $\mathrm{K}$ (molecular biology grade; New England Biolabs, Herts, UK) for 10-15 s and fixed briefly (2 min) with 4\% PFA again. Sections were incubated in 0.01\% DEPC activated PBS twice for $10 \mathrm{~min}$ each and equilibrated in $5 \mathrm{XSSC}$ (2X, $15 \mathrm{~min})$. The cross sections were prehybridized in Hybridization buffer (50\% deionized formamide [Amresco, Solon, OH], 5X SSC, $50 \mu \mathrm{g} / \mathrm{ml}$ tRNA) for $2 \mathrm{hr}$ at $61-63^{\circ} \mathrm{C}$. Hybridization reaction of S1PR2 and S1PR5a probes was carried out at $63^{\circ} \mathrm{C}$ overnight. Next day, sections were washed for 20 min with heated formamide wash buffer (hybridization buffer without tRNA and probe), 20 min with solution mix of half formamide wash buffer and half of $2 \mathrm{XSSC}$ at $60-63^{\circ} \mathrm{C}, 30 \mathrm{~min}$ in $2 \mathrm{XSSC}, 1 \mathrm{hr}$ in $2 \mathrm{X}$ SSC, $1 \mathrm{hr}$ in $0.1 \mathrm{X} \mathrm{SSC}$ and then equilibrated in Tris-buffer for $5 \mathrm{~min}$. Cross-sections were incubated in anti-DIG (digoxygenin) pAb 1:1,000 (Roche Diagnostics, Basel, Switzerland) in Tris buffer containing 0.5\% non-fat dry milk overnight at $4^{\circ} \mathrm{C}$. Cross sections were washed with Tris buffer ( 3 times, $15 \mathrm{~min}$ ) prior to color development. Staining was carried out with NBT/BCIP (Roche) for $30 \mathrm{~min}$. Sections were washed several times with Tris for 2-3 hr before mounting with Mowiol.

\section{2 | Evaluation}

Cultures were photographed at a fluorescence microscope (Zeiss Axioplan). Process length was determined and statistically evaluated using $\mathrm{NIH}$ ImageJ software.

\section{4 | RESULTS}

\subsection{Part 1 Production of rat and zebrafish M1-4}

Earlier analyses of the Rtn4 genes in fish and mammals have shown that mammalian Nogo-A/RTN4-A and zebrafish Rtn4b contain an Nterminal sequence with diagnostic motifs which was called "NogoAspecific region" (Figure 1a; Shypitsyna et al., 2011). In particular, four short motifs-termed M1-4-which possess the consensus sequence (Asp-Leu/lle-Val/Leu/lle), were found to be conserved between Rtn4b in several teleost species and in all analyzed Rtn4A of higher vertebrate species (Figure 1a). Zebrafish has three of these motifs, M1, M3, and M4, whereas the M2 region does not fully match the motif profile (Shypitsyna et al., 2011). Three of these motifs, M2-4, are within the most inhibitory Nogo-A-delta20 region of mammalian RTN4-A/Nogo-A (Oertle \& Schwab, 2003; Oertle et al., 2003; Schwab, 2010; Shypitsyna et al., 2011). M1 lies outside of the delta20 region. The recombinant zebrafish protein comprises the same region as our rat M1-4 protein and is, therefore, termed zebrafish M1-4. We expected that M1-4 in rats is as inhibitory or even more inhibitory than delta20, against which the substrate properties of zebrafish M1-4 was compared. Rat and zebrafish M1-4 as well as delta20 were recombinantly expressed as GST fusion proteins (Figure 1b). The proteins were either used with GST attached or after cleavage of the GST-tag with thrombin. Both rat M1-4 and rat Nogo-A delta20 were recognized by the mAB 11C7 (Figure 1c) (Oertle et al., 2003). Zebrafish M1-4 was recognized by pAB K1121 against Rtn4b (Figure 1b,c) (Pinzon-Olejua et al., 2014; Welte et al., 2015).

\subsection{Part 2 Inhibition of neurite growth by rat M1-4 and zebrafish M1-4 in mouse hippocampal neurons and zebrafish single cell RGCs}

To determine whether rat M1-4 inhibits neurite extension to a similar extent as Nogo-A delta20, the soluble GST-fusion proteins were applied to PDL-coated coverslips. Dissociated neurons from P1-2 mouse hippocampus and single cell zebrafish RGCs were added. Five days later, cells were fixed, stained with Phalloidin and the length of neurites was determined (Figure 2a). Neurite length was significantly reduced on GST-delta20 and rat GST-M1-4 for both mouse and zebrafish neurons, compared to GST as control (quantification from three independent experiments). GST-delta20 caused a reduction in neurite length of hippocampal neurons by $37 \%$, and rat GST-M1-4 by $64 \%$ indicating that GST-M1-4 is even more inhibitory to growing axons than GST-delta20. Surprisingly, zebrafish GST-M1-4 also caused a reduction of neurite length in hippocampal neurons by $54 \%$ (Figure 2a, b). Neurite length of single cell fish RGCs was also reduced, $24 \%$ by GST-delta20, $51 \%$ by rat GST-M1-4 and $48 \%$ by zebrafish GST-M1-4 (Figure 2a,b). Here again, rat as well as zebrafish GST-M1-4 were more inhibitory than rat GST-delta20. Thus, rat M1-4 and zebrafish M1-4 cause a reduction in neurite length in single cell cultures of mouse hippocampal and zebrafish RGC neurons. 

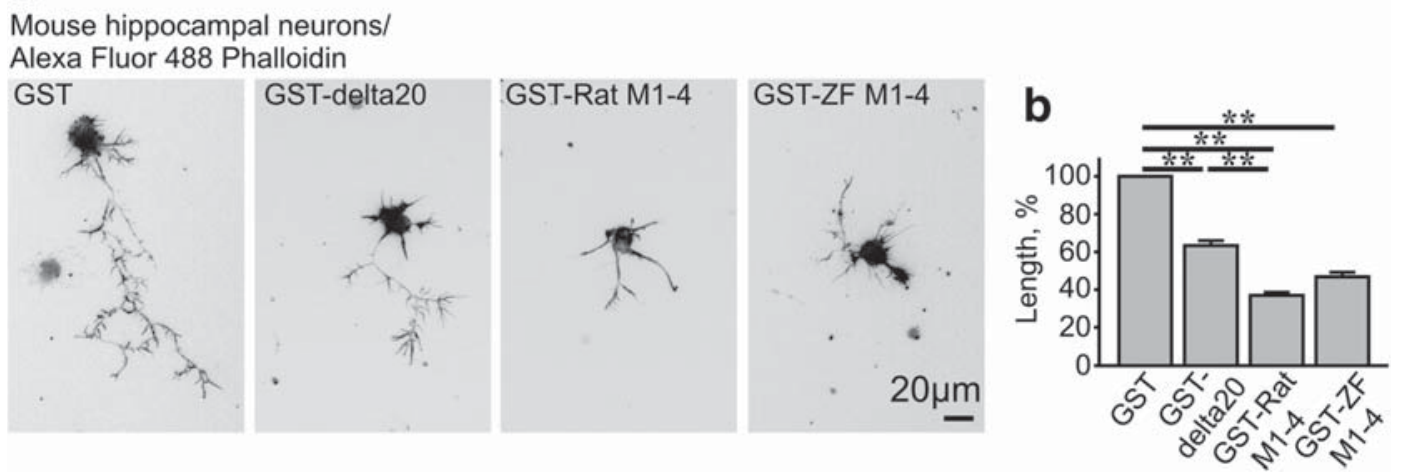

C

Zebrafish RGC/

Alexa Fluor 568 Phalloidin

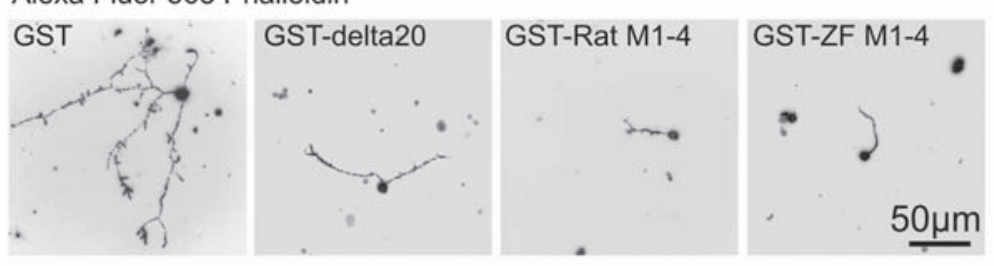

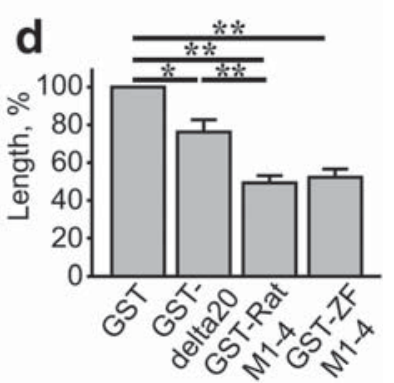

FIGURE 2 Substrate properties of Rat M1-4 and Zebrafish M1-4. Outgrowth assay. (a) Hippocampal neurons (stained by phalloidin) were plated on GST, GST-delta20, GST-Rat M1-4, and GST-Zebrafish (ZF) M1-4 and the length of the longest neurite was determined. Neurons were stained by phalloidin. Scale bar, $20 \mu \mathrm{m}$. (b) The quantification of neurite length showed that GST-delta20 compared to GST (100\%) reduced neurite length by $37 \%$. Values are $56.01 \pm 1.8 \mu \mathrm{m}$ (mean \pm SEM) with GST and $35.4 \pm 1.4 \mu \mathrm{m}$ (mean \pm SEM) with GST-delta20 as substrate, ${ }^{*} p<.05$, neurons $(\mathrm{n})=646$ and $\mathrm{n}=599$, respectively. On GST-Rat M1-4 neurite length was even more reduced than on GSTdelta20. GST-Rat M1-4 reduced length by $64 \%$ compared to GST and $42 \%$ compared to GST-delta20. Values are $56.01 \pm 1.8 \mu \mathrm{m}$ (mean \pm SEM) on GST and $20.7 \pm 0.9 \mu \mathrm{m}$ (mean \pm SEM) on GST-rat M1-4, ${ }^{*} p<.05, \mathrm{n}=646$ and $\mathrm{n}=703$, respectively. Neurite length was also reduced on GST-ZF M1-4 by $54 \%$ compared to GST. Values are $56.01 \pm 1.8 \mu \mathrm{m}$ (mean \pm SEM) on GST and $26.2 \pm 1.3 \mu \mathrm{m}$ (mean \pm SEM) on GST-ZF M1-4, ${ }^{*} p<.05$ ( $n=646$ and $n=734$ ). Neurons ( $n$ ) from three independent experiments. One-way ANOVA. Pairwise comparisons between groups (Holm-Sidak method). (c) Zebrafish single cell RGCs (stained by phalloidin) also showed reduced neurite length on GSTdelta20, GST-Rat M1-4 and GST-Zebrafish (ZF) M1-4 compared to GST alone. Scale bar: $50 \mu \mathrm{m}$. (d) The quantification showed a $24 \%$ reduction in RGC neurite length of zebrafish single cell RGCs on GST-delta20. Values are $20.2 \pm 1.3 \mu \mathrm{m}$ (mean \pm SEM) on GST and $15.4 \pm$ $1.3 \mu \mathrm{m}$ (mean \pm SEM) on GST-delta20, ${ }^{*} p<.05, \mathrm{n}=307$ and $\mathrm{n}=323$. GST-Rat M1-4 reduced neurite length by $51 \%$ compared to GST. Values are $20.2 \pm 1.3 \mu \mathrm{m}$ (mean \pm SEM) on GST and $10 \pm 0.7 \mu \mathrm{m}$ (mean \pm SEM) on GST-rat M1-4, ${ }^{*} p<.05,(\mathrm{n}=307$ and $\mathrm{n}=303)$. GST-ZF M1-4 reduced neurite length by $48 \%$. Values are $20.2 \pm 1.3 \mu \mathrm{m}$ (mean \pm SEM) on GST and $10.6 \pm 0.9 \mu \mathrm{m}$ (mean \pm SEM) on GST-ZF M1-4, ${ }^{*} p<.05,(n=307$ and $n=324$, respectively). Neurons $(n)$ from three independent experiments. One-way ANOVA. Pairwise comparisons between groups (Holm-Sidak method). Scale bar: $20 \mu \mathrm{m}$

\section{3 | Part 3 Stripe-assay}

The substrate properties of rat and zebrafish M1-4 were also evaluated in a choice assay. Proteins were applied onto Plys-coated coverslips with the aid of a special matrix (Vielmetter et al., 1990) so that protein stripes on Plys alternate with pure Plys stripes. Axons from retina explants were confronted with alternating lanes of Plys and rat M1-4, and Plys and zebrafish M1-4, respectively, and with Plys versus GST in the controls. Rat and zebrafish M1-4 proteins were used after cleavage of GST with thrombin because axons grew on both lanes when GST remained attached. Retinal explants were prepared from adult goldfish and zebrafish as well as embryonic chick retinae. Neurons of these species are known to be inhibited by rat CNS myelin, Nogo-A-delta20 and rat Nogo-66, respectively (Abdesselem et al., 2009; Bastmeyer et al., 1991; Wanner et al., 1995).
Goldfish retinal explants were used in most assays (five independent experiments, each with all substrates side by side, 50 retina explants) because the larger goldfish retina provides at least twice as many explants per retina as zebrafish retinae. The behavior of zebrafish axons was monitored pairwise on rat M1-4 versus Plys, zebrafish M14 versus Plys, and GST versus Plys, with 22 retina strips. Our assays show that the behavior of zebrafish RGCs on M1-4 resembles goldfish RGCs (see below; Figure 3).

The stripe assay with chick retinal explants was performed in four independent experiments each experiment corresponding to one retina. From each retina two explant strips per condition (rat M1-4 vs. Plys, zebrafish M1-4 vs. Plys, and GST vs. Plys) were cultured and evaluated. The quantification of the stripe assay was performed according to Vielmetter and Stuermer (1989) with category a representing no 

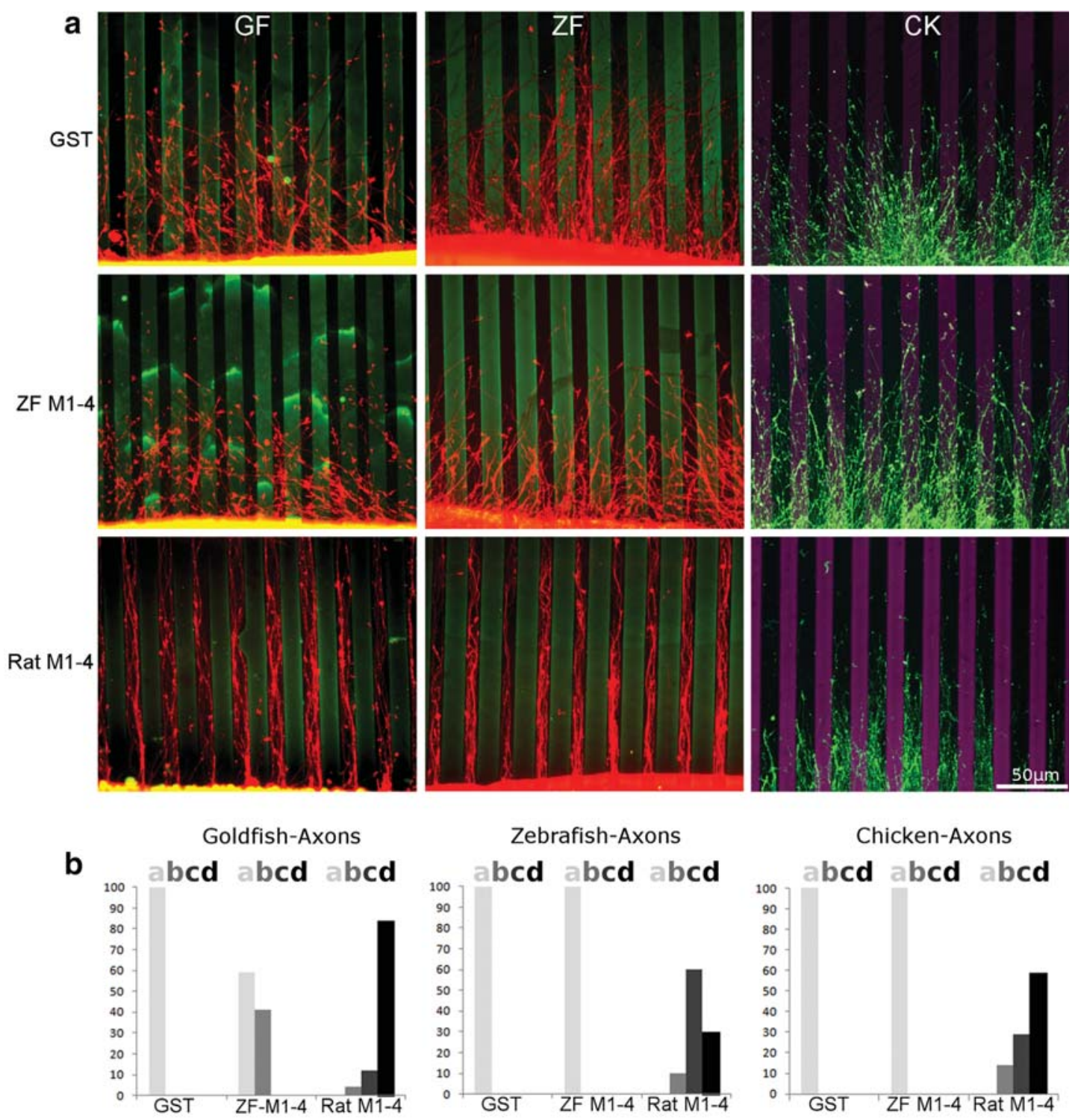

FIGURE 3 RGC axons avoid rat M1-4 but not zebrafish M1-4 in stripe assays. The substrate stripes were visualized by staining with antibodies against GST, the PAB K1121 against zebrafish M1-4, and 11C7 against Nogo-A delta20 (Rat M1-4), respectively. The axons were stained with Phalloidin. (a) Goldfish (GF) RGC axons on Rat M1-4 compared to zebrafish (ZF) M1-4. On a substrate consisting of alternating lanes of Plys with GST versus Plys-only, goldfish RGC axons from a retinal explant (to the bottom of the image) cross the striped carpet randomly. On alternating lanes of Plys with Zebrafish (ZF) M1-4 and Plys only, RGC axons cross the stripes. However, on alternating lanes of Plys plus Rat M1-4 and Plys-only, goldfish RGC axons grow preferentially on Plys and avoid Rat M1-4. The quantification shows that most (84\%) of the goldfish explants and axons extending on stripes strictly avoid Rat M1-4 (category d); $12 \%$ show growth preferentially on Plys but crossing of Rat M1-4 lanes also occurred (category c); 4\% of explants showed growth across Plys and Rat M1-4 (category b); random growth across stripes (category a) was not observed with Rat M1-4. With GST, axon growth was random (100\% category a). On ZF M1-4 axons grew randomly (category a, 59\%) or mostly random (category b, 41\%). Scale bar, $50 \mu \mathrm{m}$. (b) Zebrafish (ZF) RGC axons from retinal explants also grow randomly on a striped substrate of Plys plus GST and Plys-only. They cross stripes of Plys plus ZF M1-4 but grow preferentially on Plys stripes and avoid Rat M1-4. Quantification of the stripe assay showing that $30 \%$ of the ZF retinal explants grow strictly (category d) and 60\% preferentially on Plys stripes (category c) with Rat M1-4 in the neighboring lane, and $10 \%$ show many M1-4 stripe crossings (category b). With ZF M1-4, axons from ZF explants grow randomly across stripes (100\% category a). Random growth across stripes is also seen with GST (100\% category a). (c) Chicken (CK) RGC axons on Rat M1-4 compared to ZF M1-4. Axons from the chick retina grow on Plys stripes and avoid Rat M1-4. They grow across stripes with ZF M1-4, and stripes with GST. The explants are at the bottom of the image. Scale bar, $50 \mu \mathrm{m}$. The quantification of the stripe assays shows that $59 \%$ of axons of chicken retina explants are mostly accumulated on Plys and avoid Rat M1-4 (category d); 29\% show a preference for Plys but also can cross M1-4 stripes (category c); $12 \%$ show significant stripe crossing (category b). With ZF M1-4, 100\% of the chick retina explants show random growth of axons across stripes (category a). The same random growth was seen for stripes with Plys and GST (100\% category a) 

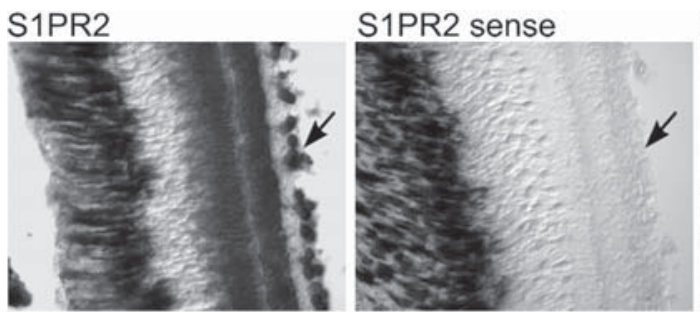

S1PR5a

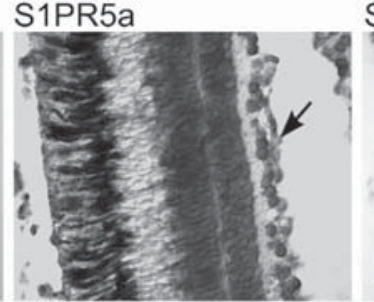

S1PR5a sense

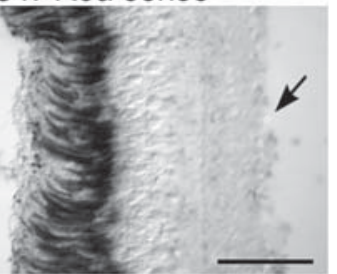

FIGURE 4 Expression of candidate Nogo-A receptors S1PR2 and S1PR5a in zebrafish RGCs. Cross sections through the zebrafish retina were subjected to in situ hybridization and show S1PR2 and S1PR5a expression in zebrafish RGCs. The sense controls (right of the pairs of images) are blank. The black structures to the left in each image represent the retinal pigment epithelium. RGCs, marked by arrows, show dark staining by the antisense probe indicative of S1PR2 and S1PR5a mRNAs. Scale bar, $50 \mu \mathrm{m}$

stripes, random growth; b, mostly random, some in stripes; c, mostly stripes, some crossing; $d$, almost all grow in stripes.

Goldfish RGC axons showed a clear preference for Plys and avoided the rat M1-4 containing stripes (Figure 3). $84 \%$ of the explants were in category $\mathrm{d}, 14 \%$ in c, $4 \%$ in b and none in a. Zebrafish RGC axons exhibited the same avoidance response with rat M1-4 as goldfish showing that goldfish and zebrafish axons are inhibited by rat M14 in the same way as with Nogo-A delta20 in earlier experiments (Abdesselem et al., 2009; Bastmeyer et al., 1991; Manns et al., 2014; Wanner et al., 1995). With zebrafish retina on rat M1-4, 30\% of the explants were in category d, $60 \%$ in c, $10 \%$ in b and $0 \%$ in a. Thus, with rat M1-4, most retina explants extended axons in stripes (Figure 3).

However, with zebrafish M1-4, goldfish RGC axons as well as zebrafish RGC axons freely crossed the stripes (Figure 3). $0 \%$ of the goldfish explants grew in stripes of categories $d$ and c, $41 \%$ fell in $b$ and $59 \%$ in a. With zebrafish retina on ZF M1-4, 0\% were in categories d, c, b and $100 \%$ were in a. With GST applied in stripes, neither goldfish nor zebrafish axons grew in stripes and instead crossed the striped substrate randomly (100\% in a). These results suggest that zebrafish M1-4-despite its similarity to rat M1-4-is growthpermissive in this type of stripe assay.

That zebrafish M1-4 might, for some reason, have become inactive is unlikely since the stripe assay employed protein from the same batches that were used in the outgrowth assay. Moreover, when cells were seeded onto stripes we observed that $31.6 \%$ less cells extended neurites on zebrafish M1-4 (and $35.4 \%$ less on rat M1-4) compared to Plys which is indicative of inhibitory substrate properties.

To test whether the difference in substrate properties of zebrafish M1-4 and rat M1-4 can also be recognized by RGC axons of warm blooded vertebrates, we exposed RGC axons from embryonic E6-7 chicken retina to the same alternating lanes of rat M1-4 and Plys, and zebrafish M1-4 and Plys, respectively. As demonstrated (Figure 3), chick RGC axons avoided the rat M1-4 containing lanes (59\% in category $\mathrm{d}, 29 \%$ in c, $12 \%$ in b and $0 \%$ in a) but freely crossed lanes with zebrafish M1-4 (100\% in category a). Likewise, growth of chicken retina explants on GST was random (100\% in category a).

The growth behavior of goldfish, zebrafish and chicken RGC axons suggests that zebrafish M1-4 is growth-permissive in this choice assay and does not cause inhibition to the same extent as rat M1-4.

\section{4 | Part 4 Zebrafish RGCs express S1PR2 and S1PR5a as candidate Nogo-A receptors}

The G-protein coupled receptor (GPCR) S1PR2 was recently identified as one of the axonal receptors that binds the Nogo-A-specific region in mammals and transmits inhibition (Kempf et al., 2014). Homologues of S1PR2 and S1PR5 were identified in zebrafish. Morpholinos against S1PR2 as well as chemical inhibitors have been used and approved to be functional in zebrafish embryos in the context of gastrulation, heart development and vascular patterning (Gu et al., 2011; Kai et al., 2008; Kupperman, An, Osborne, Waldron, \& Stainier, 2000; Mendelson, Zygmunt, Torres-Vazquez, Evans, \& Hla, 2013). We investigated whether members of this GPCR family might potentially be involved in the reaction of zebrafish RGCs to Nogo-A. Using RT-PCR we found that S1PR2 and S1PR5a are transcribed in the zebrafish retina. To verify that the receptors are expressed in RGCs, we performed in situ hybridization with probes specific for S1PR2 and S1PR5a on retina sections. Our results show that RGCs express these two receptors (Figure 4). If these receptors are relevant for the inhibition of RGC axon growth on M1-4, inhibitors and receptor-type specific MOs should allow growth of longer axons in single cell RGC cultures on these substrates. We applied MOs specific for either S1PR2 or S1PR5a to single cell RGCs growing on rat M1-4 and zebrafish M1-4, respectively. The specific MOs were used at two different concentrations ( 0.5 and $1 \mu \mathrm{M})$, in parallel to control $\mathrm{MO}$ at corresponding concentrations (Figure $5 \mathrm{a}-\mathrm{c}$ ). Both S1PR MOs caused an increase in neurite length on rat M1-4 and zebrafish M1-4 in a concentration-dependent manner (Figure 5b,c). With control MO, neurite length on M1-4 was still reduced.

The GPCR S1PR2 can be inhibited by JTE-013 and S1PR1,3- 5 by FTY-720. When outgrowth was determined in the presence of JTE013 at $5 \mu \mathrm{M}$ and FTY-720 at $1 \mu \mathrm{M}$, respectively, an increase in neurite length was observed in single cell RGCs on rat M1-4 and zebrafish M1-4 (Figure 6a,b). These results imply that S1PR2 and S1PR5a (the only other member of the family which is expressed in RGCs) are candidate Nogo-A and M1-4 receptors in zebrafish RGCs.

These results can explain why zebrafish (and goldfish) RGCs recognize mammalian myelin and Nogo-A as described in earlier studies (Bastmeyer et al., 1991; Diekmann et al., 2005). Unfortunately, it was not possible to use the receptor antagonists in the stripe assay with retinal explants at sufficiently high concentrations that would disrupt 

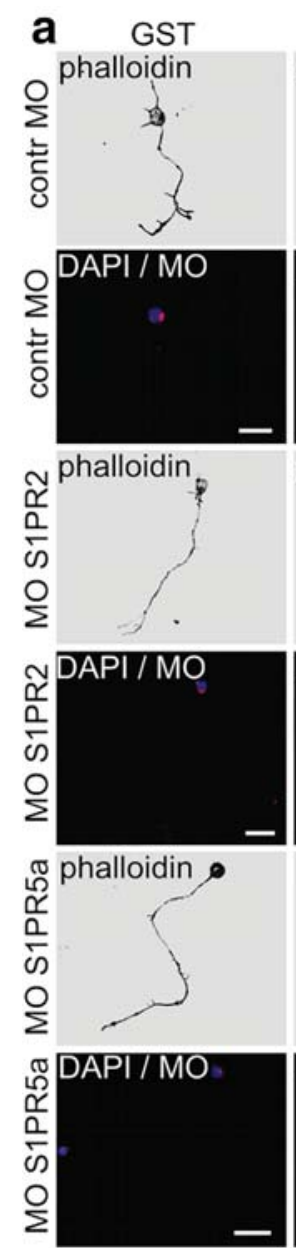

RatM1-4 phalloidin<smiles>[Te]</smiles>
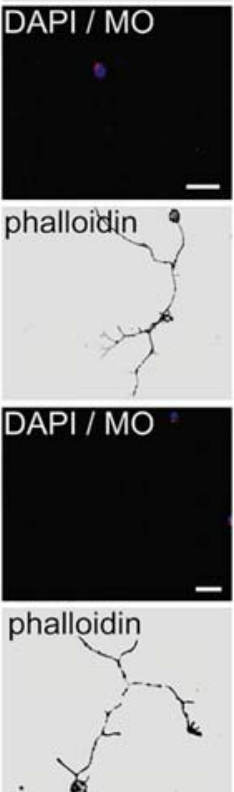

DAPI / MO

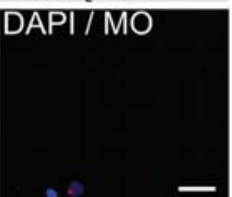

ZFM1-4

phalloidin
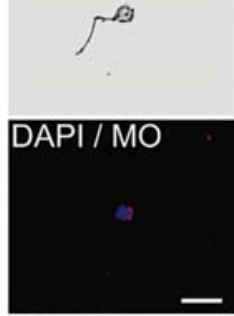

phalloidin
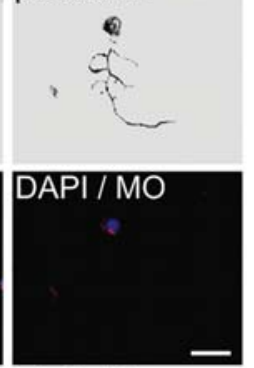

phalloidin
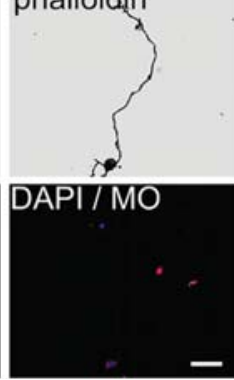

b
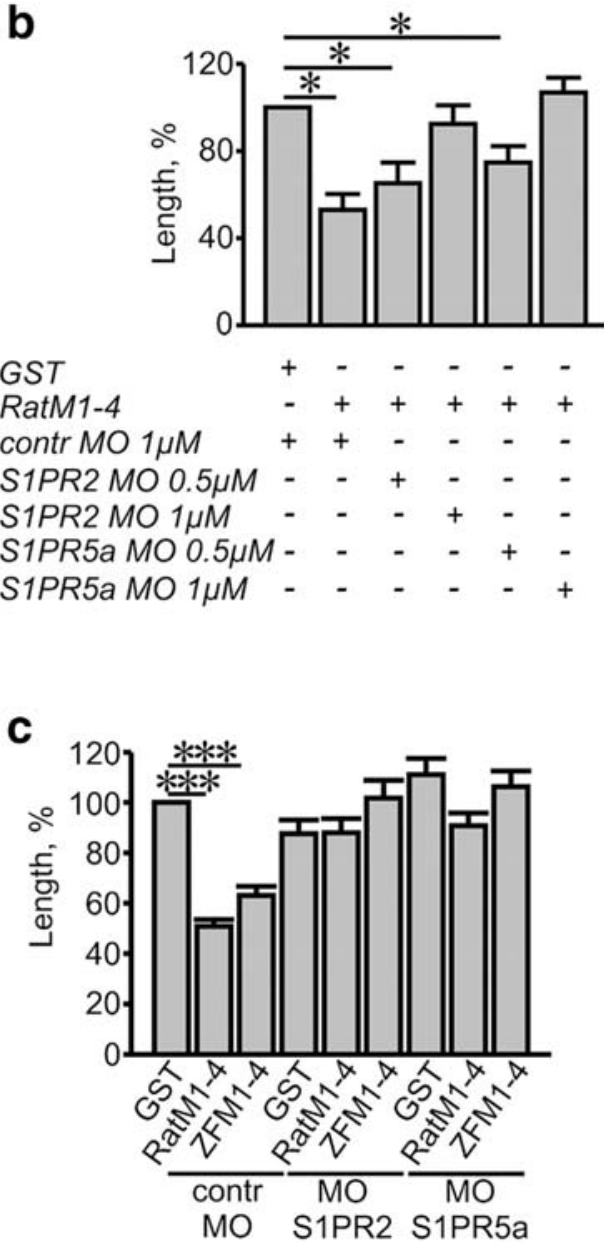

FIGURE 5 Morpholinos against S1PR2 and S1PR5a reduce the inhibitory influence of rat M1-4 and zebrafish M1-4 on RGCs. (a) Zebrafish single cell RGCs were plated on GST, Rat M1-4 or Zebrafish (ZF) M1-4. Cells were transfected with lissamine-labeled MOs against S1PR2 and S1PR5a or control MO at two concentrations of 0.5 and $1 \mu \mathrm{M}$, and stained with phalloidin to visualize cell morphology. DAPI was used to visualize the position of the nucleus (blue) and the lissamine (red) attached to the MO, right next to it. Scale bar, $40 \mu \mathrm{m}$. (b) Quantification showed that the reduction in neurite length on Rat M1-4 compared to GST was improved after downregulation of the putative Nogo receptors by MOs against S1PR2 and S1PR5a in a concentration-dependent manner (with 0.5 and $1 \mu \mathrm{M}$ MO). Neurite length was $250.6 \pm 1.9 \mu \mathrm{m}$ (mean \pm SEM) on GST with control MO and 132.7 $\pm 1.8 \mu \mathrm{m}$ on Rat M1-4 with control MO. Neurite length on Rat M14 increased to $163.6 \pm 2.3 \mu \mathrm{m}$ (mean \pm SEM) with $0.5 \mu \mathrm{M} \mathrm{S1PR2}$ and to $231.3 \pm 2.1 \mu \mathrm{m}$ (mean \pm SEM) with $1 \mu \mathrm{M}$ S1PR2, ${ }^{*} p<.05$, $\mathrm{n}=358$, $\mathrm{n}=352, \mathrm{n}=324, \mathrm{n}=346$ ). In experiments with MO against S1PR5a, neurite length was $250.6 \pm 1.9 \mu \mathrm{m}$ (mean \pm SEM) on GST with control $\mathrm{MO}$, and $132.7 \pm 1.8 \mu \mathrm{m}$ (mean \pm SEM) on Rat M1-4, and increased to $187.3 \pm 1.8 \mu \mathrm{m}$ (mean \pm SEM) with $0.5 \mu \mathrm{M}$ S1PR5a MO on Rat M1-4 and to $267.8 \pm 1.6 \mu \mathrm{m}$ (mean \pm SEM) with $1 \mu$ M S1PR5a MO $\left({ }^{*} p<.05, \mathrm{n}=358, \mathrm{n}=377, \mathrm{n}=351\right)$. Neurons $(\mathrm{n})$ from three independent experiments. One-way ANOVA. Pairwise Comparisons between Groups (Holm-Sidak method). (c) Quantification of neurite length on Rat M1-4 and ZF M1-4 with S1PR2 and S1PR5a MO at $1 \mu \mathrm{M}$. Neurite length was $601.6 \pm 5.1 \mu \mathrm{m}$ (mean \pm SEM) on GST with control MO and $303.6 \pm 1.7 \mu \mathrm{m}$ (mean $\pm S E M$ ) on Rat M1-4 with control MO, ${ }^{* * *} p<.001$, and $376.2 \pm 2.3 \mu \mathrm{m}$ (mean \pm SEM) on ZF M1-4 with control $\mathrm{MO}\left({ }^{* * *} p<.001\right)$. Neurite length was $523.4 \pm 3.4 \mu \mathrm{m}$ (mean \pm SEM) on GST with S1PR2 MO $(p=.46) ; 525.3 \pm 3.6 \mu \mathrm{m}(\mathrm{mean} \pm \mathrm{SEM})$ on Rat M1-4 with S1PR2 MO $(p=.41) ; 608.1 \pm 4.5 \mu \mathrm{m}$ (mean \pm SEM) on ZF M1-4 with S1PR2 MO $(p=.54) ; 664.2 \pm 4.1 \mu \mathrm{m}(\mathrm{mean} \pm$ SEM) on GST with S1PR5a MO $(p=.54) ; 542.8 \pm 3.3 \mu \mathrm{m}$ (mean \pm SEM) on Rat M1-4 with S1PR5a MO $(p=.73)$; and $635.4 \pm 4.1 \mu \mathrm{m}(\mathrm{mean} \pm$ SEM) on ZF M1-4 with S1PR2 MO ( $p=.89)$. ( $\mathrm{n}=314, \mathrm{n}=331, \mathrm{n}=312, \mathrm{n}=331, \mathrm{n}=380, \mathrm{n}=299, \mathrm{n}=361, \mathrm{n}=284, \mathrm{n}=328$ ). Neurons ( $\mathrm{n}$ ) from three independent experiments. One-way ANOVA. Pairwise comparisons between groups (Holm-Sidak method). Scale bar, $40 \mu \mathrm{m}$

axon growth in stripes. For instance, JTE-013 at $50 \mu \mathrm{M}$ failed to disrupt growth in stripes and precipitated when used at $100 \mu \mathrm{M}$. The receptor specific MOs could also not be used in stripe assays since MOs do not penetrate neurons when RGCs reside in retinal explants.

Several earlier studies have also emphasized that the fish visual pathway as well as fish CNS myelin was comparatively growth permissive for fish RGC axons as well as for rat RGC axons and DRGs (Bastmeyer et al., 1991; Carbonetto et al., 1987; Caroni \& Schwab, 1988; Vanselow et al., 1990; Wanner et al., 1995). How can this be reconciled with the finding that fish CNS myelin contains Rtn4b (Welte et al., 2015) whose M1-4 is apparently inhibitory in the assays with single cell neurons? This was addressed in 
a

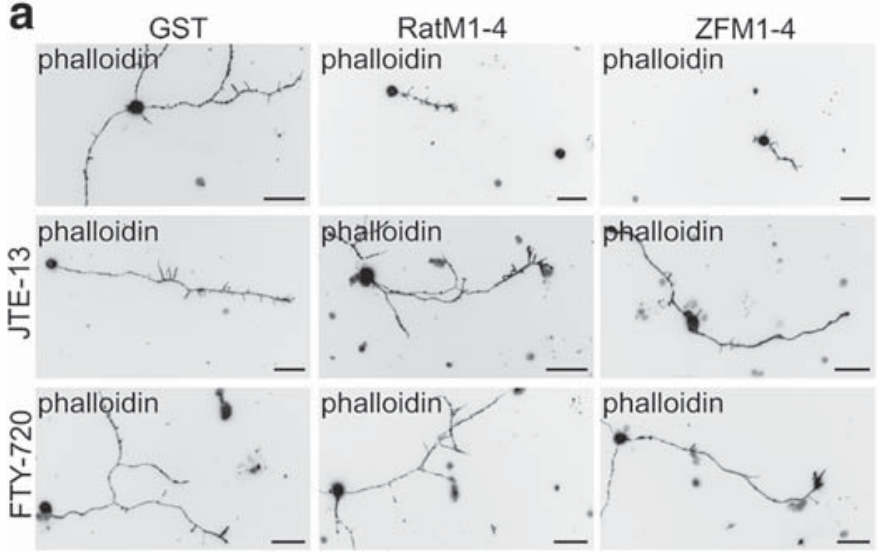

b

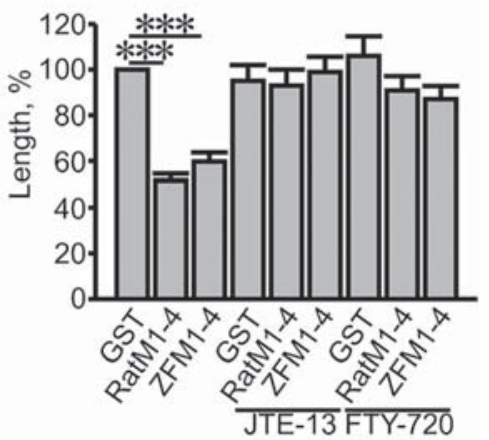

FIGURE 6 Inhibitors of S1PR2 and S1PR5a reduce the inhibitory influence of rat M1-4 and zebrafish M1-4 on RGCs. (a) Zebrafish single cell RGCs were plated on Rat M1-4, zebrafish (ZF) M1-4 and GST as control and cultured in the presence of compounds JTE-013 and FTY-720 which block S1PR2 and S1PR1,3-5, respectively. Cells were stained with phalloidin to visualize their morphology. Scale bar, 50 $\mu \mathrm{m}$. (b) Quantification showed that the inhibition of neurite length by Rat M1-4 and Zebrafish (ZF) M1-4 compared to GST was improved by JTE-013 and FTY-720 which block the potential Nogo receptors. On GST neurite length was $610.8 \pm 4.4 \mu \mathrm{m}$ (mean \pm SEM), on Rat M14 it was $313.7 \pm 1.8 \mu \mathrm{m}$ (mean \pm SEM, $\left.{ }^{* * *} p<.001\right)$ and on Zebrafish M1-4 it was $362.4 \pm 2.5 \mu \mathrm{m}$ (mean $\left.\pm S E M,{ }^{* * *} p<.001\right)$ in controls. On GST with JTE-013 neurite length was $576.8 \pm 4.6 \mu \mathrm{m}$ (mean \pm SEM, $p=.56$ ). On Rat M1-4 and with JTE-013, neurite length was $563.9 \pm$ $4.6 \mu \mathrm{m}$ (mean \pm SEM, $p=.77$ ); on zebrafish (ZF) M1-4 and JTE-013, it was 600.7 $\pm 4.4 \mu \mathrm{m}$ (mean $\pm S E M, p=.87$ ). On GST and with FTY-720, neurite length was $643.08 \pm 5.6 \mu \mathrm{m}$ (mean \pm SEM, $p=.9$ ), $551.08 \pm 4.1 \mu \mathrm{m}$ (mean \pm SEM) on Rat M1-4 with FTY-720 ( $p=.8$ ), and $529 \pm 3.7$ $\mu \mathrm{m}$ (mean \pm SEM) on ZF M1-4 and with FTY-720 ( $p=.84, \mathrm{n}=499, \mathrm{n}=522, \mathrm{n}=515, \mathrm{n}=502, \mathrm{n}=507, \mathrm{n}=531, \mathrm{n}=508, \mathrm{n}=672, \mathrm{n}=537)$. Neurons ( $\mathrm{n}$ ) from three independent experiments. One-way ANOVA. Pairwise Comparisons between Groups (Holm-Sidak method)

the next series of experiments with CNS myelin from zebrafish and rat.

\section{5 | Part 5 Neurite growth on zebrafish CNS myelin and rat CNS myelin and quantitative estimates of M1-4 (Rtn4b) in zebrafish myelin}

The substrate properties of CNS myelin and the relative amount of M1-4 in myelin and optic nerve was determined in three sets of experiments. (I) We reevaluated the substrate properties of zebrafish CNS myelin in comparison to rat CNS myelin. (II) We performed immunostainings against Rtn4b on optic nerves at 3 days after ONS to obtain an estimate of the distribution of Rtn $4 b$ at the time when axons begin to regenerate. We compared this staining to normal nerves and PO staining to visualize myelin. We also used the $A B$ against Rtn4a on sections of the same nerves since Rtn4a, more precisely its Nogo-66 domain, was found to promote rather than to inhibit RGC axon regeneration (Abdesselem et al., 2009). (III) We determined in dot blots the relative amount of Rtn4a and - $b$ in the optic nerve at 3 days after ONS and in myelin at 5 days after ONS when axons regenerate into the brain-side optic nerve. (IV) In addition, Western blots were performed with nerves at 3 days after ONS.

In the first experiment, CNS myelin was added to PDL-coated coverslips as patches of 300-600 $\mu \mathrm{m}$ in diameter, onto which either single cell zebrafish RGCs or else mouse hippocampal neurons were seeded (Figure 7a,c). Five days later, neurons were fixed and stained with Phalloidin and neurite length was quantified on myelin and on PDL next to the myelin dots as control. We also realized that there were significantly fewer cells on rat CNS myelin than on zebrafish CNS myelin as determined by DAPI-staining of the nuclei. Counts of nuclei on both substrates showed that rat CNS myelin had on average 15.5 cells per $100 \times 100 \mu \mathrm{m}$ and zebrafish CNS myelin had on average 19.2 cells per $100 \times 100 \mu \mathrm{m}$. Thus, there are $19.4 \%$ fewer cells on rat compared to zebrafish CNS myelin. Neurite length was also more strongly reduced by rat CNS myelin than zebrafish CNS myelin. Length of mouse hippocampal neurons on rat CNS myelin was $45 \%$ reduced in comparison to zebrafish CNS myelin (Figure 7c,d). RGC neurite length on rat CNS myelin was reduced by $31 \%$ compared to zebrafish CNS myelin (Figure $7 a, b)$. Even though neurite length was significantly higher on zebrafish compared to rat CNS myelin it was $41 \%$ less (hippocampal neurons) and $30 \%$ less (zebrafish single cell RGCs) than on Plys which is consistent with the notion that myelin is generally not a good substrate (Bastmeyer et al., 1991). These results confirm that CNS myelin from fish is growth permissive in comparison to rat CNS myelin, despite the fact that zebrafish CNS myelin contains M1-4 which, when offered as the sole substrate, proved to be inhibitory to single cell RGCs and hippocampal neurons (Figure 2). This suggests that the concentration of M1-4 in zebrafish myelin may be low, particularly at the time when axons regenerate in the optic nerve.

Therefore, we asked whether zebrafish Rtn $4 \mathrm{~b}$ is present to any significant extent in the path of the regenerating axons and at the time when growth cones cross the lesion site to grow toward the brain.

To address this issue we made cryosections through the zebrafish optic nerve, normal and at 3 days after ONS for immunostainings with the $A B$ against the myelin protein PO (Saul et al., 2010), and stained sections of the same nerve with the $A B$ against Rtn $4 b$ (Figure 8). The $A B$ against $P O$ showed that myelin is recognized in the normal optic nerve and is still abundant at 3 days after ONS (Figure $8 \mathrm{~d}$ ), the time 


\section{a}

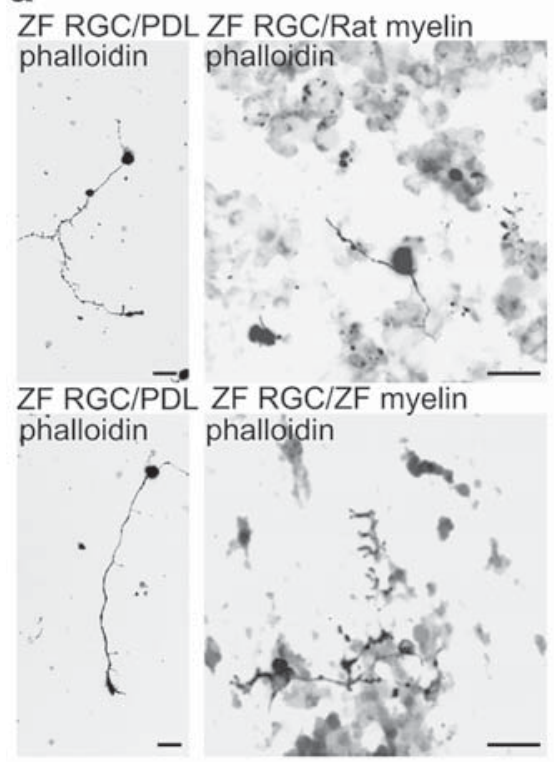

C

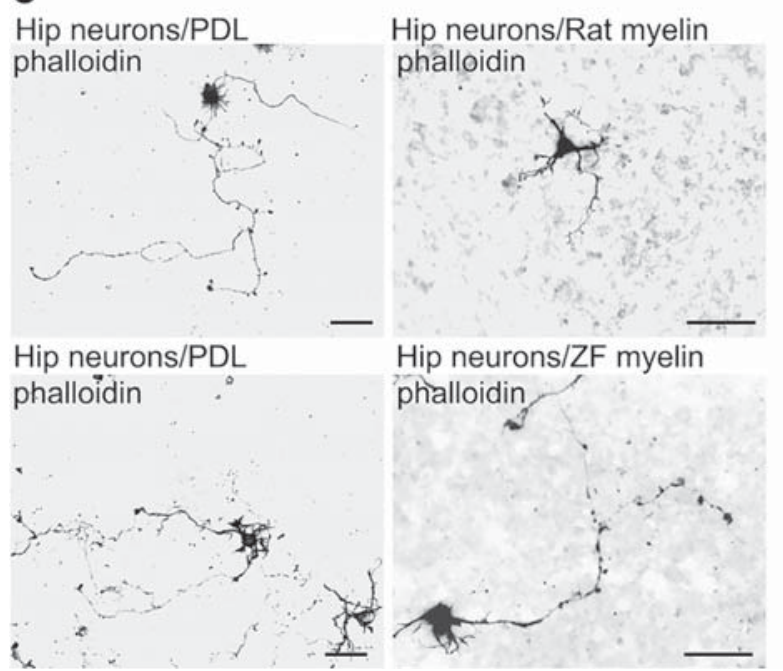

b

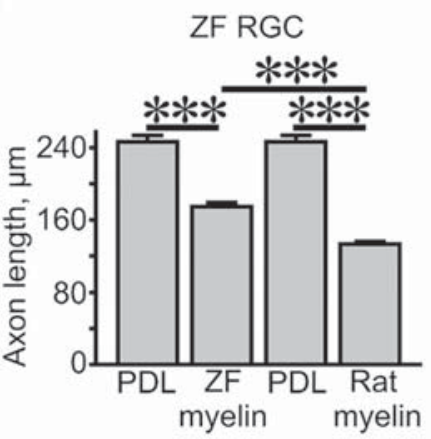

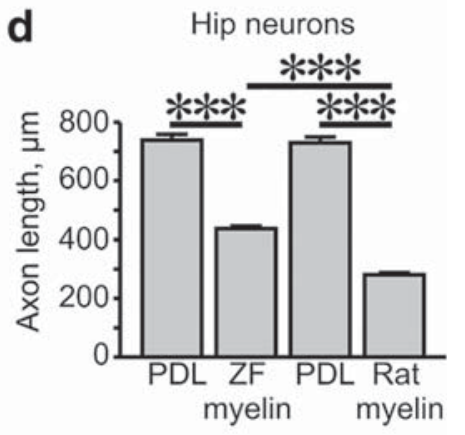

FIGURE 7 Neurite growth on CNS myelin. (a) RGCs (stained by phalloidin) reside on PDL outside of myelin patches (left) and on Rat CNS myelin or Zebrafish (ZF) CNS myelin (right), respectively. Scale bar, $40 \mu \mathrm{m}$. (b) Neurite length of ZF RGCs was significantly reduced (24\%) on rat CNS myelin compared to ZF CNS myelin. The value are $173.8 \pm 0.5 \mu \mathrm{m}$ (mean \pm SEM) on ZF CNS myelin and $132.4 \pm 0.33 \mu \mathrm{m}$ (mean \pm SEM) on Rat CNS myelin, ${ }^{* * *} p<.001, \mathrm{n}=1,438, \mathrm{n}=1,391$ ). Growth on ZF CNS myelin was $30 \%$ reduced compared to the PDL substrate surrounding the myelin. The values are $246.56 \pm 0.7 \mu \mathrm{m}$ (mean \pm SEM) on PDL and $173.8 \pm 0.5 \mu \mathrm{m}$ (mean \pm SEM) on ZF CNS myelin, ${ }^{* * *} p<.001, n=1,041, n=1,391$ ). Growth on Rat CNS myelin was $47 \%$ reduced compared to the PDL substrate surrounding the myelin. Specific values are $245.6 \pm 0.7 \mu \mathrm{m}$ (mean \pm SEM) on PDL and $132.4 \pm 0.33 \mu \mathrm{m}$ (mean \pm SEM) on Rat CNS myelin, ${ }^{* * *} p<.001$, $n=1,188, n=1,438$. Neurons ( $n$ ) from three independent experiments. Two-tailed unpaired Student's $t$-test. (c) Hippocampal neurons (Hip neurons) are depicted which reside on PDL outside of myelin patches (left) and on Rat CNS or ZF myelin (right), respectively. Scale bar, 40 $\mu \mathrm{m}$. (d) The quantification shows that neurite length of hippocampal neurons on rat CNS myelin was $35 \%$ reduced compared to growth on zebrafish CNS myelin. Specific values are 436.2 $\pm 1.7 \mu \mathrm{m}$ (mean \pm SEM) on ZF CNS myelin and $279.7 \pm 1.3 \mu \mathrm{m}$ (mean \pm SEM) on Rat CNS myelin, ${ }^{* * *} p<.001, \mathrm{n}=912, \mathrm{n}=740$ ). Growth on Zebrafish CNS myelin is $41 \%$ reduced compared to the PDL substrate surrounding the myelin. Values are: $737.8 \pm 3.9 \mu \mathrm{m}$ (mean \pm SEM) on PDL and 436.2 $\pm 1.7 \mu \mathrm{m}$ (mean \pm SEM) on ZF CNS myelin, ${ }^{* * *} p<.001, \mathrm{n}=842$, $\mathrm{n}=912$. Growth on Rat CNS myelin is $62 \%$ reduced compared to the PDL substrate surrounding the myelin. The specific values are $728.5 \pm 3.8 \mu \mathrm{m}$ (mean \pm SEM) on PDL and $279.7 \pm 1.3 \mu \mathrm{m}$ (mean $\pm S E M$ ) on Rat CNS myelin, ${ }^{* * *} p<.001, \mathrm{n}=794, \mathrm{n}=740$. Neurons ( $\mathrm{n}$ ) from three independent experiments. Two-tailed unpaired Student's $t$-test

when regenerating axons enter into the brain-side nerve (Strobel et al., 1994; Welte et al., 2015). Rtn4b staining was comparatively weak in the normal nerve and was even weaker at 3 days after ONS (Figure 8a, b). Earlier work (Welte et al., 2015) showed that Rtn4b immunostaining intensity increases at 5 and 10 days after ONS because of the arrival of regenerating axons. Rtn4b is upregulated in RGCs after ONS and 

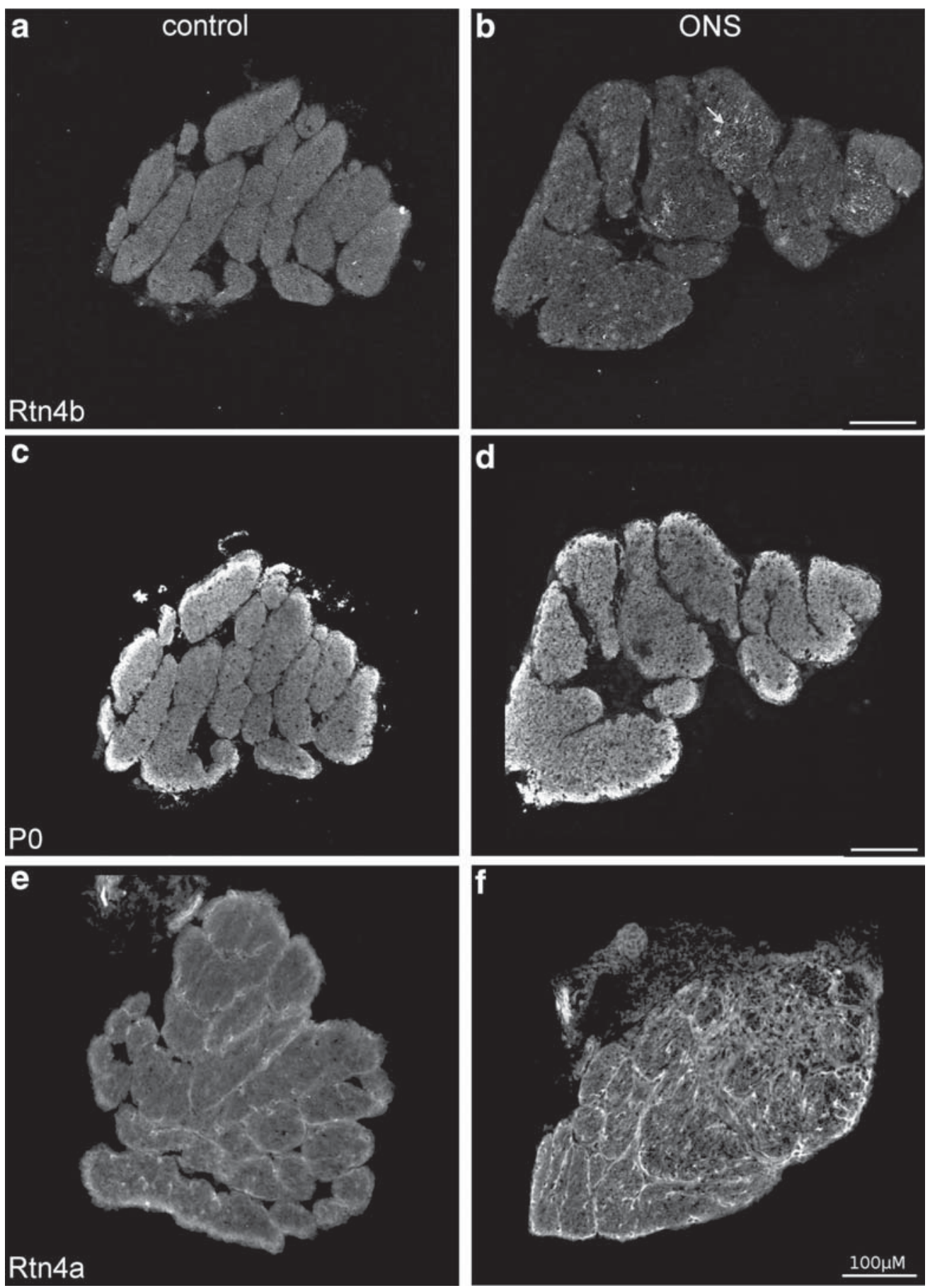

FIGURE 8 Immunostainings of zebrafish optic nerve cryo-sections show a decrease of Rtn $4 \mathrm{~b}$ after optic nerve section (ONS). Cross section through the zebrafish optic nerve, normal (control) and 3 days after ONS, were exposed to pABs against Rtn4b (a, b), Rtn4a (e, f) and PO (c, d). (a, b) Rtn4b staining is weak in the normal nerve and further decreases in the nerve after ONS The small structures with high immunostaining intensity (arrows) represent most likely the first regenerating axons (Welte et al., 2015). (c, d) Staining with anti-P0 shows myelin in the optic nerve which is abundant in the normal nerve as well as at 3 days after ONS (on consecutive sections to a and b, respectively). (e, f) Staining with pAB against Rtn4a shows a weak staining of fascicle boundaries which increases at 3 days after ONS (consistent with Abdesselem et al., 2009). Scale bar, $100 \mu \mathrm{m}$

highly expressed in regenerating axons, the first ones of which are already seen at 3 days (Figure 8). Excluding the signal of axons, the Rtn $4 \mathrm{~b}$ staining is low in the lesioned optic nerve and myelin whereas myelin protein PO remains unchanged. Rtn4b does not seem to represent a major impediment for the regenerating axons along their path through the nerve/tract. This contrasts to the situation in mammals 
a
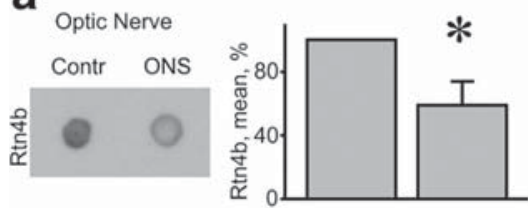

C

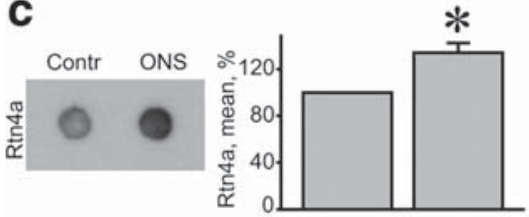

e

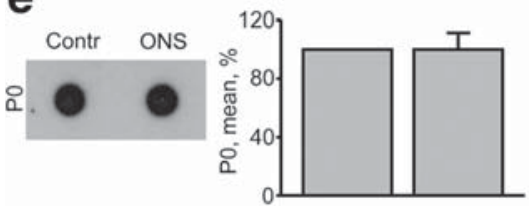

b

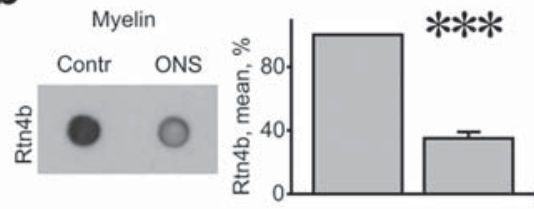

d
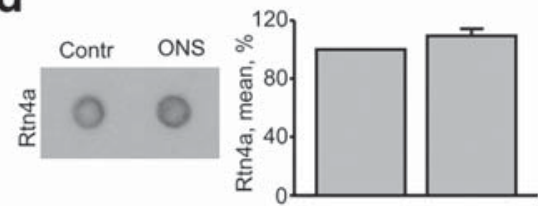

f

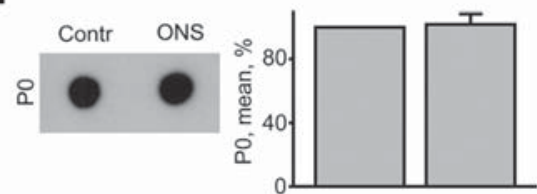

g Contr ONS

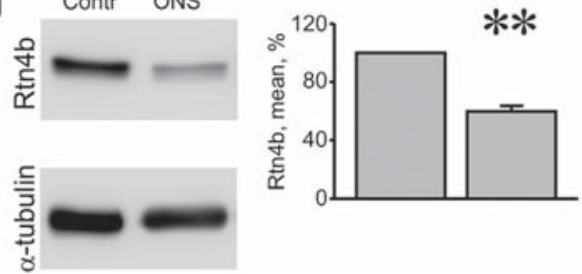

FIGURE 9 Content of Rtn4b is reduced in zebrafish optic nerve myelin after lesion. Lysates of whole optic nerves, normal and at 5 days after ONS (a, c, e), and myelin preparations obtained from the normal and lesioned optic nerve 5 days after ONS (b, d, f), were probed with specific ABs for their content of the myelin protein P0, Rtn4a and Rtn4b. The signal with the pAB against Rtn4b was significantly reduced in whole nerve extracts (a) and myelin (b) after ONS. Values are $8.8 \pm 1.0$ (mean $\pm S E M$ ) in control nerve and $5.2 \pm 1.1$ (mean $\pm S E M$ ) in nerves after ONS, ${ }^{*} p<.05(\mathrm{n}=8, \mathrm{n}=8$ ), and $8.9 \pm 1.1$ (mean \pm SEM) in control (contr) myelin and $3.1 \pm 0.3$ (mean \pm SEM) in myelin after ONS, ${ }^{* * *} p<.001$ ( $\mathrm{n}=8, \mathrm{n}=8$ ). (c) Rtn4a was significantly increased in whole optic nerve after ONS compared to control nerves (8.9 \pm 1.0 [mean $\pm S E M]$ in control nerve and $12.0 \pm 0.7$ (mean \pm SEM) in the optic nerve after ONS, ${ }^{*} p<.05, n=8, n=8$ ). (d) Rtn4a was slightly increased in myelin fractions after ONS, with specific values of $10.4 \pm 0.5$ (mean $\pm S E M$ ) in control myelin and $11.4 \pm 0.5$ (mean \pm SEM) in myelin after ONS, $p=.19(\mathrm{n}=8, \mathrm{n}=8$ ). (e) The signals obtained with $\mathrm{pAB}$ against PO were equal in whole nerve extracts with and without ONS. Specific values are $42.3 \pm 4.8$ (mean \pm SEM) in control nerves and $42.2 \pm 4.8$ (mean \pm SEM) in optic nerve after ONS, $p=.99$ ( $n=8$, $\mathrm{n}=8$ ). (f) PO was equal in myelin with and without ONS. It was $30.0 \pm 1.6$ (mean $\pm S E M$ ) in myelin from control nerves and $30.6 \pm 1.9$ (mean \pm SEM) in myelin after ONS $(p=.84, n=8, n=8)$. The quantification in histograms next to the dot blots show results of four independent experiments. Two-tailed unpaired Student's $t$-test. (g) Western blots with lysates from normal nerves (Contr) and nerves at 3 days after ONS also demonstrate a $40.7 \%$ decrease in Rtn4b in the brain-side optic nerve/tract. Alpha-tubulin served as loading control. Specific values (intensity of protein band compared to tubulin) are: $6.95 \pm 0.37$ (mean \pm SEM) in control and $4.12 \pm 0.31$ (mean \pm SEM) at 3 days after ONS. The quantification in the histogram next to the blot show results of three independent experiments $\left({ }^{* *} p<.005\right)$

where RTN4-A/Nogo-A is strong in optic nerve myelin, remains strong after lesion (Hunt, Coffin, Prinjha, Campbell, \& Anderson, 2003; Pernet, Joly, Christ, Dimou, \& Schwab, 2008) and impairs axon regeneration.

Comparison of stainings by $A B$ against Rtn $4 b$ with $A B$ against Rtn4a-which is growth promoting-shows that Rtn4a, in contrast to Rtn $4 b$, is increased after ONS (Figure $8 e, f$ ) and is mostly in fascicle boundaries and axons as demonstrated earlier (Abdesselem et al., 2009).

To obtain a better estimate on the Rtn4b content in myelin, dot blots were performed with fractions highly enriched in myelin from nerves at 5 days after ONS and with whole nerve homogenates at 3 days after ONS. This was compared to Rtn4b in myelin and whole nerve homogenates of normal nerves. Dot blots were used since they allow a better estimate of the amount of a specific protein such as Rtn4b per volume tissue (especially myelin). To guarantee that the same amount of myelin and nerve homogenate was used in separate dot blots, optical density of the probes was measured prior to application. In addition, whole nerves, normal and at 3 days after ONS, were prepared for Western blots. Samples were probed with ABs against PO and Rtn4b. The dot blots in Figure 9 show strong signals for PO both in the normal nerve and at 3 days after ONS (Figure 9e). Rtn4b in optic nerve myelin and homogenate was 62 and 42\%, respectively, reduced at 5 and 3 days after ONS compared to myelin and homogenate of the normal nerve (Figure 9a,b). Rtn4a, however, was present in the normal 
and regenerating optic nerve and was $34 \%$ increased in the whole nerve homogenate and $9 \%$ in optic nerve myelin at 3 and 5 days after ONS (Figure 9c,d) consistent with the immunostainings (Figure 8) and earlier results (Abdesselem et al., 2009). The reduction of Rtn $4 \mathrm{~b}$ in the 3 days ONS nerve also became evident in Western blots showing a $40.7 \%$ reduction of Rtn $4 b$ after lesion compared to the normal control (Figure 9g).

The results led to the conclusion that the concentration of Rtn $4 \mathrm{~b}$ is low in optic nerve myelin and optic nerve total at the time when axons regenerate into the brain side optic nerve. Thus, even if M1-4 of Rtn $4 b$ is inhibitory when offered as substrate to RGCs, it seems not to be present to any significant extent in the path of regenerating axons after optic nerve lesion. Moreover, consistent with earlier (Bastmeyer et al., 1991; Wanner et al., 1995) and present results, zebrafish CNS myelin substrate properties are significantly better in terms of axon growth support than rat CNS myelin for fish RGCs as well as mouse hippocampal neurons.

\section{5 | DISCUSSION}

The identification of Rtn $4 \mathrm{~b}$ as the zebrafish homolog of Nogo-A/ RTN4A and the resemblance of motifs M1-4 in zebrafish Rtn4b and rat RTN4A, has led us to re-assess the question whether inhibitors of axon growth are present in fish CNS myelin and optic nerve and affect axon regeneration. Zebrafish M1-4 proved to be slightly less inhibitory than rat M1-4 (and Nogo-A delta20) when used as the sole substrate for single cell neuronal cultures. However, zebrafish M1-4 was not or less inhibitory to RGC axons than rat M1-4 in the stripe assay with retinal explants from goldfish, zebrafish, and chick. This suggests that zebrafish M1-4 is inhibitory or permissive in a context dependent manner. The reaction of axons to M1-4 differs depending on whether they emerge from retinal explants or from single cell RGCs and dissociated hippocampal neurons. Our data suggest furthermore, that the zebrafish RGCs possess S1PR2 and S1PR5a as receptors (or members of a receptor complex) for the inhibition by substrate-associated M1-4 proteins. Most importantly, however, zebrafish optic nerve contains apparently very little Rtn4b, particularly after ONS. Therefore, zebrafish Rtn4b seems to be no major obstacle for axon regeneration.

\section{$5.1 \mid$ Zebrafish M1-4 and Rtn4b}

In mammals, Nogo-A/RTN4-A (and thus M1-4) is rich in CNS myelin in the path of severed axons (Pernet et al., 2008) and blocks their growth in vivo and in vitro (Schwab, 2010). Not so in fish. Myelin fractions from the zebrafish CNS were a significantly better substrate for neurite growth of single cell RGCs and hippocampal neurons than rat CNS myelin. This result is in accordance with earlier data (Bastmeyer et al., 1991; Caroni \& Schwab, 1988; Wanner et al., 1995). However, zebrafish optic nerve myelin, normal and particularly at 3 and 5 days after ONS, contains apparently very little Rtn $4 b$, as demonstrated by immunostainings of optic nerve sections and Western and dot blot analysis of myelin fractions of the lesioned optic nerve. Other myelin proteins, in particular MBP (Welte et al., 2015) and PO, persist in myelin for at least 10 days. Thus, myelin is abundant at the time when regenerating axons pass through the nerve (Strobel \& Stuermer, 1994; Welte et al., 2015) but potentially inhibitory Rtn4b/M1-4 is low when regenerating axons arrive. Curiously, Rtn4a, the paralogue of Rtn4b (Shypitsyna et al., 2011), is known to be growth supportive in fish (Abdesselem et al., 2009) and increases in the lesioned nerve.

The present findings are in agreement with many earlier reports showing the relative growth permissive properties of the fish optic nerve and myelin compared the nonpermissive and inhibitory properties of mammalian CNS myelin (Bastmeyer et al., 1991; Caroni \& Schwab, 1988; Wanner et al., 1995). Beyond the previous knowledge our findings now show that potentially inhibitory M1-4 is expressed in zebrafish but at very low levels in the lesioned optic nerve. Consistent with this finding is the earlier observation that retinal growth cones in the regenerating optic nerve are, in fact, closely associated with myelin debris (Strobel \& Stuermer, 1994). At later stages of optic nerve regeneration, that is, at 10 days, Rtn $4 b$ increases in the zebrafish optic nerve. This increase is, however, associated with regenerating axons from RGCs which massively upregulate Rtn $4 b$ after optic nerve lesion (Welte et al., 2015). Thus, the growth inhibitory activity of zebrafish M1-4 for single cell RGCs and hippocampal neurons in vitro seems to be irrelevant for regenerating axons in vivo due to the relative low abundance of Rtn4b. And the neuron-intrinsic properties (Fawcett, 2006; Stuermer et al., 1992) allow RGCs in vivo to grow even if they encounter zebrafish Rtn4b M1-4. The RGCs in the fish retina upregulate a number of transcription factors and proteins which promote growth (Elsaeidi, Bemben, Zhao, \& Goldman, 2014; Paschke, Lottspeich, \& Stuermer, 1992; Saul et al., 2010; Stuermer, 2010; Stuermer et al., 1992; Veldman, Bemben, Thompson, \& Goldman, 2007; Vielmetter et al., 1990; Welte et al., 2015) including Rtn4b in the ER (Welte et al., 2015). This upregulation is also found in RGCs in retinal explants (which are derived from nerve transected retinae) so that the RGCs in explants have the same growth supportive neuron-intrinsic properties. In explants in vitro (as well as in vivo) RGCs retain contact with neighboring neurons and glial cells and thus obtain trophic support which dissociated neurons are deprived of. The different conditions of RGCs could explain the difference in the reaction to M1-4 in the outgrowth and stripe assay. Chick RGC axons are equally well-equipped with neuronintrinsic growth promoting molecules since they derive from embryonic neurons at the stage of maximal axon growth.

That dissociated neurons in single cell neuronal cultures are more sensitive to inhibitors than axons from retinal explants is supported by the fact, that the effective concentration of M1-4 was 100 times less in the outgrowth compared to the stripe assay.

Rtn4 proteins are structural proteins of the ER (Rämö et al., 2016; Shibata et al., 2008), and Rtn4b was accordingly seen in the ER of fish oligodendrocytes in vitro (Welte et al., 2015) and in fish RGCs. The fact that RGCs after lesioning the optic nerve upregulate Rtn4b and that downregulation impairs axon regeneration is consistent with the notion that Rtn4 proteins in the ER play an important role for the regrowth of lesioned axons (Manns et al., 2014; Merianda et al., 2009; Welte et al., 2015). Axon regrowth requires a massive increase in protein synthesis. 
The function of Rtn $4 b$ in oligodendrocytes and myelin may as well be ER associated. Whether zebrafish Rtn4b is brought to the cell surface as was reported for Nogo-A in mammals (Schwab, 2010) requires more sophisticated immunostaining methods (Dodd et al., 2005). Still, Rtn4b could become accessible to regenerating axons in the degenerating myelin debris that lies in their path through the nerve and tract (Strobel \& Stuermer, 1994). However, the difference in immunostainings obtained with the antibody against mammalian RTN4A/Nogo-A in myelin of lesioned optic nerves and spinal cord of mice and rats (Pernet et al., 2008) is striking. Zebrafish Rtn4b decreases in the brain-side optic nerve/tract after lesion so that zebrafish Rtn4b is by far less abundant and no impediment for regenerating axons. The immunostaining intensity of RTN4A/Nogo-A in the optic nerve and spinal cord is high proximal as well as distal to the lesion for many days (Hunt et al., 2003; Pernet et al., 2008). This is consistent with the inability of mammalian axons to regenerate into the myelin/Nogo-A-containing nerves.

\section{2 | Nogo receptors}

The present and former experiments with rat myelin and Nogo-A and fish axons implied that fish RGCs should possess the Nogo-A receptor (s) (Bastmeyer et al., 1991). This receptor was recently identified. In mammals, the GPCR S1PR2 is the signal transducing element of a receptor complex for Nogo-A delta20 (Kempf et al., 2014; Thiede-Stan et al., 2015). Our in situ hybridization experiments show that zebrafish RGCs express the homologue of S1PR2, as well as S1PR5a which is another member of the family. That both are candidate Nogo-A and Rtn4b (M1-4) receptors is concluded from results with S1PR2- and S1PR5a-specific morpholinos and chemical inhibitors. The finding that receptor subtype-specific morpholinos increase neurite length on rat and zebrafish M1-4 and counteract inhibition in a concentration dependent manner and that the same increase in neurite length is obtained with S1PR2 specific and S1PR1,3-5 chemical inhibitors suggest that regenerating growth cones use these receptor(s) for Nogo-Adelta20 and M1-4. But as in mammals, S1PR2 and S1PR5a are not only receptors for M1-4, Nogo-A delta20 and M1-4 in zebrafish Rtn4b, but were identified as receptors for sphingosine- 1 phosphate (Lee et al., 1998; Mendelson et al., 2013) with many functions in blood vessel and heart development.

Even though more work is needed to better characterize the zebrafish Nogo receptors, their expression and function complies with the fact that zebrafish express Rtn4b as homologue of Nogo-A/RTN4a, and thus one of the ligands for these receptors. It remains to be determined at which stage these receptors are needed during axon growth in normal development and regeneration.

\section{3 | Evolutionary aspects of axon regeneration and Nogo-like inhibitors}

Nogo-A is widely expressed in the mammalian and avian CNS and present not only on oligodendrocytes and in myelin but also on the neuronal surface including dendrites and axons. Nogo-A is thought to act as a repressor of synaptic plasticity (Petrinovic et al., 2013; Schwab, 2010; Zemmar et al., 2014) in mammals and regulator of axon fasciculation in chick (Petrinovic et al., 2010). Reptiles (like the lizard Gallotia gallotis) also possess Nogo-A in CNS myelin but curiously, the lizard retinal axons do not recognize it as inhibitor probably because of different signal transduction properties of the Nogo receptors (Lang, Monzon-Mayor, Bandtlow, \& Stuermer, 1998; Lang, Romero-Aleman, Dobson, Santos, \& Monzon-Mayor, 2016).

Nogo-A homologues were also discovered in amphibians like Xenopus (Klinger et al., 2004). In the adult animal, axons fail to regenerate in the spinal cord, where Nogo-A is heavily expressed. Yet in the optic nerve, where Xenopus RGC axons successfully regenerate, myelin disappears and oligodendrocytes dedifferentiate during the stage at which cut axons regrow toward the brain. Instead, immature astrocyte-like cells form a growth supportive scaffold that promotes RGC axon regeneration (Bohn \& Reier, 1985; Lang, Ankerhold, \& Stuermer, 1997; Lang, Rubin, Schwab, \& Stuermer, 1995; Lang \& Stuermer, 1996).

Our present work in zebrafish, as representative of teleosts, shows the fish strategy of successful optic nerve regeneration: RGC axons in vitro are sensitive to Nogo-A and possess the relevant receptors; the potentially inhibitory M1-4 of Rtn $4 \mathrm{~b}$ is present but in comparatively small amounts in optic nerve myelin and further decreases after lesion.

Moreover, fish optic nerve oligodendrocytes dedifferentiate after lesion (Ankerhold \& Stuermer, 1999) and glia cells express growth promoting molecules on the surface (Abdesselem et al., 2009; Ankerhold, Leppert, Bastmeyer, \& Stuermer, 1998; Hirsch, Cahill, \& Stuermer, 1995). This and the unique neuron-intrinsic properties of fish RGCs showing upregulation of so many growth- associated molecules (Saul et al., 2010; Stuermer, 2010; Stuermer et al., 1992) which favor axon growth seems to encourage the elongation of regenerating axons through the nerve which is reflected by the free crossing of zebrafish M1-4 in the stripe assay. In other words, different vertebrate classes use species-specific strategies to ensure success of axon regeneration. The environment (CNS myelin and oligodendrocytes) which regenerating zebrafish axons encounter is not growth permissive because NogoA homologues are not expressed but because Rtn $4 b$ is less inhibitory than Nogo-A and reduced in quantity.

\section{ACKNOWLEDGMENT}

This work was supported by grants from the DFG to C.A.O.S. (grant numbers Stu 112/23-1,2)

\section{CONFLICT OF INTEREST}

The authors declare no conflict of interest.

\section{AUTHOR CONTRIBUTIONS}

All authors had full access to all the data in the study and take responsibility for the integrity of the data and the accuracy of the data analysis. Study concept and design: C.A.O.S. Acquisition of data: CW, MW, VB, GK, AS, APO, M Weschenfelder. Analysis and interpretation of data: CS, MB, VB, CW, M Weschenfelder. Drafting of 
the manuscript: C.A.O.S. and CW. Critical revision of the manuscript for important intellectual content: C.A.O.S., CW, VB, and MB. Statistical analysis: VB, CW. Obtained funding: C.A.O.S. Administrative, technical, and material support: MW. Study supervision: C.A.O.S.

\section{REFERENCES}

Abdesselem, H., Shypitsyna, A., Solis, G. P., Bodrikov, V., \& Stuermer, C. A. (2009). No Nogo66- and NgR-mediated inhibition of regenerating axons in the zebrafish optic nerve. Journal of Neuroscience, 29(49), 15489-15498. doi:10.1523/JNEUROSCI.3561-09.2009

Ankerhold, R., Leppert, C. A., Bastmeyer, M., \& Stuermer, C. A. (1998). E587 antigen is upregulated by goldfish oligodendrocytes after optic nerve lesion and supports retinal axon regeneration. Glia, 23(3), 257270. doi:10.1002/(SICI)1098-1136(199807)23:3 < 257::AID-GLIA8 > 3.0.CO;2-1

Ankerhold, R., \& Stuermer, C. A. (1999). Fate of oligodendrocytes during retinal axon degeneration and regeneration in the goldfish visual pathway. Journal of Neurobiology, 41(4), 572-584. doi:10.1002/(SICI) 1097-4695(199912)41:4 < 572::AID-NEU12 > 3.0.CO;2-8

Bai, Q., Sun, M., Stolz, D. B., \& Burton, E. A. (2011). Major isoform of zebrafish PO is a $23.5 \mathrm{kDa}$ myelin glycoprotein expressed in selected white matter tracts of the central nervous system. Journal of Comparative Neurology, 519(8), 1580-1596. doi:10.1002/cne.22587

Bastmeyer, M., Beckmann, M., Schwab, M. E., \& Stuermer, C. A. (1991). Growth of regenerating goldfish axons is inhibited by rat oligodendrocytes and CNS myelin but not but not by goldfish optic nerve tract oligodendrocytelike cells and fish CNS myelin. Journal of Neuroscience, 11(3), 626-640. Retrieved from http://www.ncbi.nlm.nih. gov/pubmed/2002357

Bodrikov, V., Solis, G. P., \& Stuermer, C. A. (2011). Prion protein promotes growth cone development through reggie/flotillin-dependent $\mathrm{N}$-cadherin trafficking. Journal of Neuroscience, 31(49), 18013-18025.

Bohn, R. C., \& Reier, P. J. (1985). Retrograde degeneration of myelinated axons and re-organization in the optic nerves of adult frogs (Xenopus laevis) following nerve injury or tectal ablation. Journal of Neurocytology, 14(2), 221-244. Retrieved from http://www.ncbi.nlm.nih.gov/ pubmed/4045505

Carbonetto, S., Evans, D., \& Cochard, P. (1987). Nerve fiber growth in culture on tissue substrata from central and peripheral nervous systems. Journal of Neuroscience, 7(2), 610-620. Retrieved from http:// www.ncbi.nlm.nih.gov/pubmed/3819825

Caroni, P., \& Schwab, M. E. (1988). Two membrane protein fractions from rat central myelin with inhibitory properties for neurite growth and fibroblast spreading. Journal of Cell Biology, 106(4), 1281-1288. Retrieved from http://www.ncbi.nlm.nih.gov/pubmed/ 3360853

Colman, D. R., Kreibich, G., Frey, A. B., \& Sabatini, D. D. (1982). Synthesis and incorporation of myelin polypeptides into CNS myelin. Journal of Cell Biology, 95(2 Pt 1), 598-608. Retrieved from http://www.ncbi. nlm.nih.gov/pubmed/6183276

Diekmann, H., Klinger, M., Oertle, T., Heinz, D., Pogoda, H. M., Schwab, M. E., \& Stuermer, C. A. (2005). Analysis of the reticulon gene family demonstrates the absence of the neurite growth inhibitor Nogo-A in fish. Molecular Biology and Evolution, 22(8), 1635-1648. doi:10.1093/ molbev/msi158

Dodd, D. A., Niederoest, B., Bloechlinger, S., Dupuis, L., Loeffler, J. P., \& Schwab, M. E. (2005). Nogo-A, -B, and -C are found on the cell surface and interact together in many different cell types. Journal of Biological Chemistry, 280(13), 12494-12502. doi:10.1074/jbc.M411827200
Elsaeidi, F., Bemben, M. A., Zhao, X. F., \& Goldman, D. (2014). Jak/ Stat signaling stimulates zebrafish optic nerve regeneration and overcomes the inhibitory actions of Socs 3 and Sfpq. Journal of Neuroscience, 34(7), 2632-2644. doi:10.1523/JNEUROSCI.389813.2014

Fawcett, J. W. (2006). Overcoming inhibition in the damaged spinal cord. Journal of Neurotrauma, 23(3-4), 371-383. doi:10.1089/neu.2006. 23.371

Fournier, A. E., GrandPre, T., \& Strittmatter, S. M. (2001). Identification of a receptor mediating Nogo-66 inhibition of axonal regeneration. Nature, 409(6818), 341-346. doi:10.1038/35053072

Gu, Y., Forostyan, T., Sabbadini, R., \& Rosenblatt, J. (2011). Epithelial cell extrusion requires the sphingosine-1-phosphate receptor 2 pathway. Journal of Cell Biology, 193(4), 667-676. doi:10.1083/jcb.201010075

Hirsch, S., Cahill, M. A., \& Stuermer, C. A. (1995). Fibroblasts at the transection site of the injured goldfish optic nerve and their potential role during retinal axonal regeneration. Journal of Comparative Neurology, 360(4), 599-611. doi:10.1002/cne.903600405

Hunt, D., Coffin, R. S., Prinjha, R. K., Campbell, G., \& Anderson, P. N. (2003). Nogo-A expression in the intact and injured nervous system. Molecular and Cellular Neuroscience, 24(4), 1083-1102. Retrieved from http://www.ncbi.nlm.nih.gov/pubmed/14697671

Kai, M., Heisenberg, C. P., \& Tada, M. (2008). Sphingosine-1-phosphate receptors regulate individual cell behaviours underlying the directed migration of prechordal plate progenitor cells during zebrafish gastrulation. Development, 135(18), 3043-3051. doi:10.1242/dev.020396

Kempf, A., Tews, B., Arzt, M. E., Weinmann, O., Obermair, F. J., Pernet, V., \& Schwab, M. E. (2014). The sphingolipid receptor S1PR2 is a receptor for Nogo-a repressing synaptic plasticity. PLoS Biology, 12 (1), e1001763. doi:10.1371/journal.pbio.1001763

Klinger, M., Diekmann, H., Heinz, D., Hirsch, C., Hannbeck von Hanwehr, S., Petrausch, B., \& Stuermer, C. A. (2004). Identification of two NOGO/RTN4 genes and analysis of Nogo-A expression in Xenopus laevis. Molecular and Cellular Neuroscience, 25(2), 205-216. doi: 10.1016/j.mcn.2003.09.021

Kupperman, E., An, S., Osborne, N., Waldron, S., \& Stainier, D. Y. (2000). A sphingosine-1-phosphate receptor regulates cell migration during vertebrate heart development. Nature, 406(6792), 192-195. doi: $10.1038 / 35018092$

Lang, D. M., Ankerhold, R., \& Stuermer, C. A. O. (1997). Axonal regeneration in the fish and amphibian CNS - Myelin-associated neurite growth inhibitors and adaptive plasticity of glial cells. Cell Biology and Pathology of Myelin, 4, 299-308. Retrieved from <Go to ISI >:// WOS:A1997BJ39H00029

Lang, D. M., Monzon-Mayor, M., Bandtlow, C. E., \& Stuermer, C. A. (1998). Retinal axon regeneration in the lizard Gallotia galloti in the presence of CNS myelin and oligodendrocytes. Glia, 23(1), 61-74. doi:10.1002/(SICI)1098-1136(199805)23:1 < 61::AID-GLIA6 > 3.0. $\mathrm{CO} ; 2-7$

Lang, D. M., Romero-Aleman, M. D., Dobson, B., Santos, E., \& MonzonMayor, M. (2016). Nogo-A does not inhibit retinal axon regeneration in the lizard Gallotia galloti. Journal of Comparative Neurology. 525(4), 936-954. doi:10.1002/cne.24112

Lang, D. M., Rubin, B. P., Schwab, M. E., \& Stuermer, C. A. (1995). CNS myelin and oligodendrocytes of the Xenopus spinal cord-but not optic nerve-are nonpermissive for axon growth. Journal of Neuroscience, 15(1 Pt 1), 99-109. Retrieved from http://www.ncbi.nlm.nih. gov/pubmed/7823155

Lang, D. M., \& Stuermer, C. A. (1996). Adaptive plasticity of Xenopus glial cells in vitro and after CNS fiber tract lesions in vivo. Glia, 18(2), 92- 
106. doi:10.1002/(SICI)1098-1136(199610)18:2 < 92::AID-GLIA2 > 3.0. CO;2-E

Lee, M. J., Van Brocklyn, J. R., Thangada, S., Liu, C. H., Hand, A. R., Menzeleev, R., \& Hla, T. (1998). Sphingosine-1-phosphate as a ligand for the G protein-coupled receptor EDG-1. Science, 279(5356), 15521555. Retrieved from http://www.ncbi.nlm.nih.gov/pubmed/ 9488656

Mandemakers, W. J., \& Barres, B. A. (2005). Axon regeneration: It's getting crowded at the gates of TROY. Current Biology, 15(8), R302305. doi:10.1016/j.cub.2005.04.002

Manns, R., Schmandke, A., Schmandke, A., Jareonsettasin, P., Cook, G., Schwab, M. E., \& Keynes, R. (2014). Protein synthesis dependence of growth cone collapse induced by different Nogo-A-domains. PLoS One, 9(1), e86820. doi:10.1371/journal.pone.0086820

Matsui, T., Raya, A., Callol-Massot, C., Kawakami, Y., Oishi, I., RodriguezEsteban, C., \& Izpisua Belmonte, J. C. (2007). miles-apart-Mediated regulation of cell-fibronectin interaction and myocardial migration in zebrafish. Nature Clinical Practice. Cardiovascular Medicine, 4 Suppl 1, S77-82. doi:10.1038/ncpcardio0764

Mendelson, K., Zygmunt, T., Torres-Vazquez, J., Evans, T., \& Hla, T. (2013). Sphingosine 1-phosphate receptor signaling regulates proper embryonic vascular patterning. Journal of Biological Chemistry, 288(4), 2143-2156. doi:10.1074/jbc.M112.427344

Merianda, T. T., Lin, A. C., Lam, J. S., Vuppalanchi, D., Willis, D. E., Karin, N., \& Twiss, J. L. (2009). A functional equivalent of endoplasmic reticulum and Golgi in axons for secretion of locally synthesized proteins. Molecular and Cellular Neuroscience, 40(2), 128-142. doi: 10.1016/j.mcn.2008.09.008

Oertle, T., \& Schwab, M. E. (2003). Nogo and its paRTNers. Trends in Cell Biology, 13(4), 187-194.

Oertle, T., van der Haar, M. E., Bandtlow, C. E., Robeva, A., Burfeind, P., Buss, A., \& Schwab, M. E. (2003). Nogo-A inhibits neurite outgrowth and cell spreading with three discrete regions. Journal of Neuroscience, 23(13), 5393-5406.

Paschke, K. A., Lottspeich, F., \& Stuermer, C. A. (1992). Neurolin, a cell surface glycoprotein on growing retinal axons in the goldfish visual system, is reexpressed during retinal axonal regeneration. Journal of Cell Biology, 117(4), 863-875. Retrieved from http://www.ncbi.nlm. nih.gov/pubmed/1577862

Pernet, V., Joly, S., Christ, F., Dimou, L., \& Schwab, M. E. (2008). Nogo-A and myelin-associated glycoprotein differently regulate oligodendrocyte maturation and myelin formation. Journal of Neuroscience, 28 (29), 7435-7444. doi:10.1523/JNEUROSCI.0727-08.2008

Petrinovic, M. M., Duncan, C. S., Bourikas, D., Weinman, O., Montani, L., Schroeter, A., \& Schwab, M. E. (2010). Neuronal Nogo-A regulates neurite fasciculation, branching and extension in the developing nervous system. Development, 137(15), 2539-2550. doi:10.1242/ dev.048371

Petrinovic, M. M., Hourez, R., Aloy, E. M., Dewarrat, G., Gall, D., Weinmann, O., \& Schwab, M. E. (2013). Neuronal Nogo-A negatively regulates dendritic morphology and synaptic transmission in the cerebellum. Proceedings of the National Academy of Sciences of the United States of America, 110(3), 1083-1088. doi:10.1073/ pnas.1214255110

Pinzon-Olejua, A., Welte, C., Abdesselem, H., Malaga-Trillo, E., \& Stuermer, C. A. (2014). Essential roles of zebrafish rtn4/Nogo paralogues in embryonic development. Neural Development, 9(1), 8. doi:10.1186/1749-8104-9-8

Rämö, O., Kumar, D., Gucciardo, E., Joensuu, M., Saarekas, M., Vihinen, H., \& Jokitalo, E. (2016). NOGO-A/RTN4A and NOGO-B/RTN4B are simultaneously expressed in epithelial, fibroblast and neuronal cells and maintain ER morphology. Scientific Reports, 6, 35969. doi: 10.1038/srep35969

Rosen, H., Gonzalez-Cabrera, P. J., Sanna, M. G., \& Brown, S. (2009). Sphingosine 1-phosphate receptor signaling. Annual Review of Biochemistry, 78, 743-768. doi:10.1146/annurev.biochem.78.072407.103733

Saul, K. E., Koke, J. R., \& Garcia, D. M. (2010). Activating transcription factor 3 (ATF3) expression in the neural retina and optic nerve of zebrafish during optic nerve regeneration. Comparative Biochemistry and Physiology Part A: Molecular \& Integrative Physiology, 155(2), 172182. doi:10.1016/j.cbpa.2009.10.042

Schwab, M. E. (2010). Functions of Nogo proteins and their receptors in the nervous system. Nature Reviews Neuroscience, 11(12), 799-811. doi:10.1038/nrn2936

Shibata, Y., Voss, C., Rist, J. M., Hu, J., Rapoport, T. A., Prinz, W. A., \& Voeltz, G. K. (2008). The reticulon and DP1/Yop1p proteins form immobile oligomers in the tubular endoplasmic reticulum. Journal of Biological Chemistry, 283(27), 18892-18904. doi:10.1074/jbc. M800986200

Shypitsyna, A., Malaga-Trillo, E., Reuter, A., \& Stuermer, C. A. (2011). Origin of Nogo-A by domain shuffling in an early jawed vertebrate. Molecular Biology and Evolution, 28(4), 1363-1370. doi:10.1093/mol$\mathrm{bev} / \mathrm{msq} 313$

Strobel, G., \& Stuermer, C. A. (1994). Growth cones of regenerating retinal axons contact a variety of cellular profiles in the transected goldfish optic nerve. Journal of Comparative Neurology, 346(3), 435-448. doi:10.1002/cne.903460307

Stuermer, C. A. (2010). The reggie/flotillin connection to growth. Trends in Cell Biology, 20(1), 6-13. doi:10.1016/j.tcb.2009.10.003

Stuermer, C. A., Bastmeyer, M., Bahr, M., Strobel, G., \& Paschke, K. (1992). Trying to understand axonal regeneration in the CNS of fish. Journal of Neurobiology, 23(5), 537-550. doi:10.1002/neu.480230508

Thiede-Stan, N. K., \& Schwab, M. E. (2015). Attractive and repulsive factors act through multi-subunit receptor complexes to regulate nerve fiber growth. Journal of Cell Science, 128(14), 2403-2414. doi: 10.1242/jcs.165555

Thiede-Stan, N. K., Tews, B., Albrecht, D., Ristic, Z., Ewers, H., \& Schwab, M. E. (2015). Tetraspanin-3 is an organizer of the multi-subunit Nogo-A signaling complex. Journal of Cell Science, 128(19), 35833596. doi:10.1242/jcs.167981

Vanselow, J., Schwab, M. E., \& Thanos, S. (1990). Responses of regenerating rat retinal ganglion cell axons to contacts with central nervous myelin in vitro. European Journal of Neuroscience, 2(2), 121-125.

Veldman, M. B., Bemben, M. A., Thompson, R. C., \& Goldman, D. (2007). Gene expression analysis of zebrafish retinal ganglion cells during optic nerve regeneration identifies KLF6a and KLF7a as important regulators of axon regeneration. Developmental Biology, 312(2), 596612. doi:10.1016/j.ydbio.2007.09.019

Vielmetter, J., Stolze, B., Bonhoeffer, F., \& Stuermer, C. A. (1990). In vitro assay to test differential substrate affinities of growing axons and migratory cells. Experimental Brain Research, 81(2), 283-287. Retrieved from http://www.ncbi.nlm.nih.gov/pubmed/2397757

Vielmetter, J., \& Stuermer, C. A. (1989). Goldfish retinal axons respond to position-specific properties of tectal cell membranes in vitro. $\mathrm{Neu}$ ron, 2(4), 1331-1339.

Wanner, M., Lang, D. M., Bandtlow, C. E., Schwab, M. E., Bastmeyer, M., \& Stuermer, C. A. (1995). Reevaluation of the growth-permissive substrate properties of goldfish optic nerve myelin and myelin proteins. Journal of Neuroscience, 15(11), 7500-7508. Retrieved from http:// www.ncbi.nlm.nih.gov/pubmed/7472501 
Welte, C., Engel, S., \& Stuermer, C. A. (2015). Upregulation of the zebrafish Nogo-A homologue, Rtn4b, in retinal ganglion cells is functionally involved in axon regeneration. Neural Development, 10, 6. doi:10.1186/ s13064-015-0034-x

Weschenfelder, M., Weth, F., Knoll, B., \& Bastmeyer, M. (2013). The stripe assay: Studying growth preference and axon guidance on binary choice substrates in vitro. Methods in Molecular Biology, 1018, 229-246. doi:10.1007/978-1-62703-444-9_22

Zemmar, A., Weinmann, O., Kellner, Y., Yu, X., Vicente, R., Gullo, M., \& Schwab, M. E. (2014). Neutralization of Nogo-A enhances synaptic plasticity in the rodent motor cortex and improves motor learning in vivo. Journal of Neuroscience, 34(26), 8685-8698. doi:10.1523/ JNEUROSCI.3817-13.2014 